\title{
Photoionization Models of the Inner Gaseous Disk of the Herbig Be Star BD+65 1637
}

\author{
P. Patel ${ }^{1}$, T. A. A. Sigut ${ }^{1}$ and J. D. Landstreet ${ }^{2}$ \\ Accepted for publication in The Astrophysical Journal \\ Department of Physics and Astronomy, The University of Western Ontario, \\ London, Ontario Canada N6A $3 K^{7}$ \\ ppatel54@uwo.ca
}

\begin{abstract}
We attempt to constrain the physical properties of the inner, gaseous disk of the Herbig Be star BD+65 1637 using non-LTE, circumstellar disk codes and observed spectra (3700 to 10,500 $\AA$ ) from the ESPaDOnS instrument on CFHT. The photoionizing radiation of the central star is assumed to be the sole source of input energy for the disk. We model optical and near-infrared emission lines that are thought to form in this region using standard techniques that have been successful in modeling the spectra of Classical Be stars. By comparing synthetic line profiles of hydrogen, helium, iron and calcium with the observed line profiles, we try to constrain the geometry, density structure, and kinematics of the gaseous disk. Reasonable matches have been found for all line profiles individually; however, no disk density model based on a single powerlaw for the equatorial density was able to simultaneously fit all of the observed emission lines. Amongst the emission lines, the metal lines, especially the Ca II IR triplet, seem to require higher disk densities than the other lines. Excluding the CaII lines, a model in which the equatorial disk density falls as $10^{-10}\left(R_{*} / R\right)^{3} \mathrm{~g} \mathrm{~cm}^{-3}$ seen at an inclination of $45^{\circ}$ for a $50 \mathrm{R}_{*}$ disk provides reasonable matches to the overall line shapes and strengths. The Ca II lines seem to require a shallower drop off as $10^{-10}\left(R_{*} / R\right)^{2} \mathrm{~g} \mathrm{~cm}^{-3}$ to match their strength. More complex disk density models are likely required to refine the match to the $\mathrm{BD}+651637$ spectrum.
\end{abstract}

Subject headings: stars: pre-main sequence, stars: variable: T Tauri, Herbig Ae/Be, stars: individual (BD+65 1637), stars: emission, Be, accretion disks, line: profiles

\section{Introduction}

Herbig Ae/Be (hereafter HAeBe) stars are premain sequence $\mathrm{A}$ or B-type stars with emission lines and an excess in their infrared spectral energy distributions (SEDs). The emission lines, particularly the Balmer series of hydrogen, and the infrared excess can be attributed to circumstellar dust and gas, the likely remnants of the star formation phase (Herbig 1960; Finkenzeller \& Mundt 1984 Waters \& Waelkens 1998). Circumstellar dust distinguishes HAeBe stars from the Classical

\footnotetext{
${ }^{1}$ Center For Planetary Science \& Exploration, The University of Western Ontario, London, Ontario N6A 3K7

${ }^{2}$ Armagh Observatory, Armagh, Northern Ireland, UK
}

Be stars ${ }^{1}$ whose infrared excess is solely due to free-free emission from the ionized, dust-free gas in a circumstellar decretion disk (Rivinius et al. 2013).

Being the precursors to the debris disks, such as those around $\beta$ Pictoris and Vega, HAeBe stars make interesting subjects for studying disk physics, as well as for understanding disk evolution in pre-main sequence stars (Perez \& Grady 1997). HAeBe stars are also an important link between low and high mass star formation. High

\footnotetext{
${ }^{1}$ Classical Be stars are B-type, main-sequence stars that show, or have once shown, one or more Balmer emission lines in their spectrum.
} 
mass, O-type stars form at the centers of very dense clusters and involve complex environments. The formation of such stars is currently poorly understood (Larson 2003). In addition, such stars spend their pre-main sequence life in a deeply embedded state before becoming optically visible as main-sequence objects, hence depriving us of the opportunity to observe the early phase of star formation (Zinnecker \& Yorke 2007). In contrast, Herbig Be stars (HBe hereafter), despite forming in complex and dense environments, may become optically visible just before they reach the Zero Age Main Sequence (ZAMS) as they spend comparatively longer in the pre-main sequence (PMS, hereafter) phase compared to their higher mass counterparts. This not only aids in the understanding of the formation process of intermediate mass HBe stars, but can also help to bridge the understanding of the star formation process between low mass $\mathrm{T}$ Tauri star: $\mathrm{S}^{2}$ and high mass, O-type stars.

Knowledge of the physical conditions in the disks around HAeBe stars has been steadily increasing. The disk material is inherited by the star from its parent molecular cloud. Near-IR and millimeter interferometric observations (see the review by Kraus (2015) and references therein) have been instrumental in providing strong evidence for circumstellar disks around HAeBe stars. The disk can extend to 100s of AUs and disk temperatures can vary from a few 10 s $\mathrm{K}$ to 1000 s K (Dullemond \& Monnier 2010). These disks have been studied extensively at far-infrared and millimeter wavelengths for cooler dust species such as PAHs (Polycyclic Aromatic Hydrocarbons), iron oxide grains, and silicates (crystalline and amorphous) to understand the evolution of these species in the disk (Waters \& Waelkens 1998). Near and mid-infrared wavelengths have been used to study molecular gas and warm dust in regions closer to the star.

Vink et al. (2002), Mottram et al. (2007), and Vink (2015) have shown that there exists a difference in polarization between Herbig Ae

\footnotetext{
${ }^{2} \mathrm{~T}$ Tauri stars are pre-main sequence stars, with masses less than $2.5 \mathrm{M}_{\odot}$, which show Balmer emission lines in their spectrum and an excess in their infrared SEDs, interpreted as gas and dust in form of a circumstellar disk. The most massive $\mathrm{T}$ Tauri stars will later become main-sequence Atype stars.
}

(hereafter HAe) and HBe stars, especially early B-type stars. Vink et al. (2002) first noticed that while all HAe stars show intrinsic linear polarization consistent with magnetospheric accretion, most $\mathrm{HBe}$ stars show line-depolarization which is consistent with disk accretion. HAeBe stars show photometric variability ranging from days to months to years (Herbig 1960; Finkenzeller \& Mundt 1984), and various models such as nonradial pulsation, accretion, etc, have been suggested for their occurrence (Catala 1994). Van den Ancker et al. (1998) and Mendigutía et al. (2011) have shown that HAe stars show large to moderate variations in magnitude $\left(>2^{m} .5\right)$ while HBe stars show low to moderate variations $\left(<0^{m} .5\right)$. The differences between the variations has been suggested to be due to different accretion mechanisms. In addition, Cauley \& Johns-Krull I (2014) studied HeI $(\lambda 10830)^{3}$ in a sample of HAeBe stars and noticed that HBe stars show blue shifted absorption features while HAe stars show both blue and red shifted absorption features. This difference indicates that HBe stars show little evidence of infalling material, while HAe's show a higher level of mass flow activity, suggesting the action of different mechanisms. A recent study by Fairlamb et al. (2015) of UV excess of a large set of HAeBe stars showed that early-type HBe stars cannot be modeled successfully using the magnetospheric accretion, again hinting of an alternate mode of accretion.

Alecian et al. (2008, 2013) studied a large set of HAeBe stars and found that less than $10 \%$ of them show evidence of large scale magnetic fields. Possible causes for a lack of large scale magnetic fields for the HAe and HBe stars are either a very small convective core or completely radiative envelope, neither of which generate large scale magnetic fields (Alecian 2014). Accretion via a magnetosphere is fairly well established for T Tauri stars (see reviews by Bouvier et al. (2007) and Gómez de Castro (2013)) but whether or not the same mechanism applies to HAeBe stars is still an open question. With the differences in the polarizations and the lack of large scale magnetic fields, the mechanism at work for accretion in HBe stars is still a mystery.

\footnotetext{
${ }^{3}$ All wavelengths are in $\AA$ in this study unless otherwise stated.
} 


\section{Disk Structure}

\subsection{Atomic Gaseous Disk}

Close to the star, one expects regions with high temperatures that would destroy any dust and create a dust-free zone. Further away, the temperature is cool enough for dust to exist, and in the region favorable for dust, one either finds a thick wall of dust, sometimes called a dust rim, or a smooth transition to dusty disk at the dust sublimation radius (see Dullemond \& Monnier (2010) for a review). Monnier et al. (2005) suggest that if an optically thin gas exists in the dust-free zone, a wall of dust is expected as direct radiation from the star will enhance the scale height of the dust rim or puff up the inner disk wall. If an optically thick gas exists, then the transition to dust is smoother.

As dust evaporates above $\sim 1500 \mathrm{~K}$, the portion of the disk closest to the star is likely to be completely gaseous due to high temperatures resulting from the star's UV radiation. Depending on the distance from the central star and the stellar radiation, HBe disks can be divided into atomic gas, molecular gas, and dust. The expected structure of such a disk is illustrated in Figure 1. The inner gaseous region extends from, perhaps, $0.1 \mathrm{AU}$ to a few AUs $\left(\sim 100 R_{*}\right)$ in size, and temperatures can reach to 1000 s K. Optical and Near-IR (NIR, hereafter) atomic lines are used to study this hot, atomic gaseous region. The disk region beyond the gaseous disk receiving direct radiation from the star are expected to have a thin surface layer with atomic gas; hence, the emission seen in the spectrum can arise from a large extended area. In the region beyond the atomic gaseous disk, where the temperatures are cooler, molecular gas is expected. As the temperature decreases, the molecular gas condenses into dust and a mixture of warm dust and molecular gas is expected. NIR molecular emission lines and NIR/Mid-IR (MIR, hereafter) interferometry is used to understand the structure of this region. Beyond this region, cooler gas and dust are detected using millimeter and submillimeter interferometry. The region beyond the atomic gaseous disk can range anywhere in size from 0.1 to 100 s of AUs, including the dusty disk. The outer, dusty region of the disk is well studied, but currently very little is known about the inner, gaseous region.
Several studies have suggested that HBe stars can be compared to Classical Be stars (see Hamann \& Persson (1992a); Mottram et al. (2007)) in their circumstellar disk and structure. Hillenbrand et al. (1992) noticed that some of the HAeBe stars in their sample show a small IR excess, comparable to that seen in Classical Be stars. Like early-type HBe stars, Classical Be stars are known to show depolarization (see Rivinius et al. (2013)). In addition, similarities such as the $\mathrm{H} \alpha$ equivalent width distribution, fast rotation speeds (i.e. high $v$ sini), photometric variability, and slow outflow velocities further connect the Classical $\mathrm{Be}$ and HBe stars (Böhm \& Balona 2000). Hence, using models that have been well established for Classical Be stars would make a good first step in understanding the inner, atomic gaseous disks of HBe stars.

\subsection{Molecular Gas \& Dust Disk}

Several studies of molecular gas using tracers such as $\operatorname{Br} \gamma$ ( Mendigutía et al. (2011); Calvet et al. (2004)) and CO (e.g. Ilee et al. (2014, 2013); Wheelwright et al. (2010)) have been conducted, probing the relatively warm region of the disk where these emission lines are thought to originate. Ilee et al. (2014) studied CO overtone emission and strongly suggests that the emission originates from a small gaseous disk inside the dust sublimation radius that follows Keplerian rotation. This hints at the process of classical disk accretion where material is thought to be transfered from the disk directly onto the star through an equatorial disk.

Monnier \& Milan-Gabet (2002) and MillanGabet et al. (2007) noticed that most HBe stars have a smaller dust sublimation radius $\left(R_{\text {rim }}\right)$ than predicted by the luminosity-size $\left(R_{\text {rim }} \propto\right.$ $L_{*}^{1 / 2}$ ) relationship developed for the T Tauri and HAe stars. Monnier \& Milan-Gabet (2002) suggest that the smaller size of the gaseous disk around HBe stars may be due to optically thick gas absorbing the UV radiation, allowing the dust to exist closer to the star. Eisner et al. (2004) was able to fit an inner rim model to a flat disk with optically thick gas to $2.2 \mu \mathrm{m}$ observations for higher mass stars. Alonso-Albi et al. (2009) showed that the disks around HBe stars are 5-10x less massive than those of lower mass counterpart HAe and $\mathrm{T}$ Tauri stars and proposed that strong UV radiation 


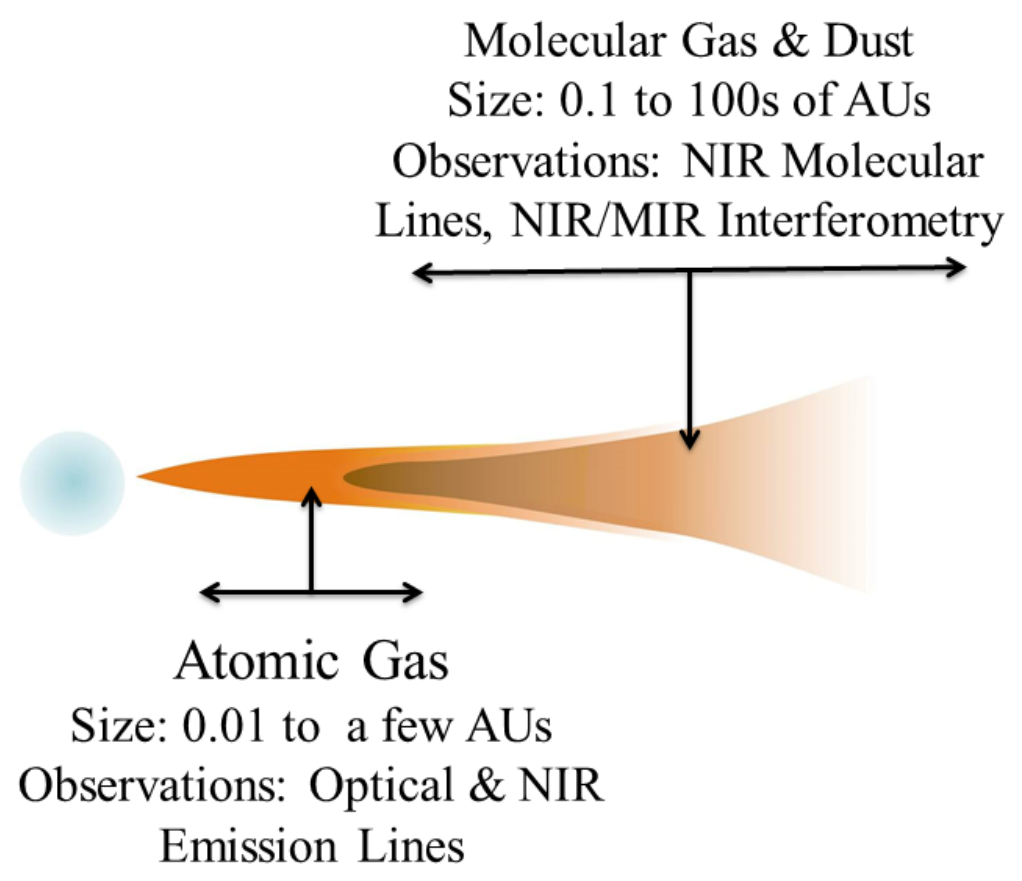

Fig. 1.- Inferred structure of the disk around HBe stars. Close to the star, partially ionized, atomic gas is expected. The size of this region may extend anywhere from 0.01 AU to a few AUs. This region can be studied using optical and near-infrared spectral lines. As the temperatures cool in the disk, molecular gas and dust is expected. The size of this outer disk ranges from 0.1 to 100s of AUs. The transition between atomic and molecular gas occurs at around a few AUs. The molecular gas and dust found beyond the atomic gaseous disk is represented by brown, and this region can be studied using variety of different methods such as molecular lines and NIR/MIR interferometry. It is important to note that this image is not to scale. Image Credit: Ami Patel 
from the hot, central star evaporates and disperses the gas, leaving behind a small dusty disk. Finally, the gas in the circumstellar disk is seen to follow Keplerian rotation, as found by Mannings \& Sargent (1997, 2000) using millimeter interferometric measurements.

\subsection{Emission Lines}

By definition, all HAeBe stars show emission lines in their spectra which can be used to trace the structure and processes in the disk. Hamann \& Persson (1992a) show that the spectra of HAeBe stars are very similar to those of $\mathrm{T}$ Tauri stars; however, they note that the similarity of the spectra does not mean that the formation mechanism of the lines is the same. The higher stellar temperatures of the HAeBe stars can change both the mechanisms and the extent to which the gas is excited due to the intense stellar radiation field experienced by the disk gas.

Only a few studies have qualitatively modeled the permitted emission lines seen the visible and NIR part of the spectrum. Cohen \& Kuhi (1979), Finkenzeller \& Mundt (1984), Hamann \& Persson (1992a) and Böhm \& Catala (1995) investigated emission lines such as $\mathrm{H} \alpha$, the Ca II IR triplet $(\lambda 8498, \lambda 8542 \& \lambda 8662)$ and several Fe II lines and interpreted them as revealing chromospheric and wind activity in these stars. More recently, He I ( $\lambda$ 10830) has been used as a tracer of mass flow activity by Cauley \& Johns-Krull (2014). In addition to permitted emission lines, forbidden emission lines are found to be present in HAeBe stars, and Corcoran \& Ray (1997) have shown that these lines may arise from the winds and outflows.

Finkenzeller \& Mundt (1984) showed that the $\mathrm{H} \alpha$ line in $\mathrm{HAeBe}$ stars can be divided into three groups based on the line profile morphology, namely single-peaked, double-peaked and PCygni line profiles. More than $50 \%$ of the stars studied showed a double-peaked $\mathrm{H} \alpha$ profile, while the rest were divided equally into single-peaked and P-Cygni profiles. Finkenzeller \& Jankovics (1984) note that all Balmer lines exhibit the same line shape as $\mathrm{H} \alpha$, with the strength of the line decreasing from $\mathrm{H} \alpha$ to $\mathrm{H} \gamma$. $\mathrm{H} \alpha$ has been widely used for wind diagnostics studies of Herbig Ae/Be stars (Finkenzeller \& Mundt 1984, Corcoran \& Ray 1998) and polarization (Vink et al. 2002
Vink 2015).

Hamann \& Persson (1992a) and Hamann \& Perrson (1992b) investigated the Ca II triplet emission in HAeBe stars: $71 \%$ of their HBe stars showed the Ca II IR triplet lines in emission. They showed that if the excitation is same in all the stars, Ca II requires denser and/or thicker regions in hotter stars (see their Figure 8). They also noted that due to high stellar temperatures and the double-peaked line profiles, the formation of the Ca II lines would happen away from stellar surface in a disk like those possessed by Classical Be stars and in a very small, ring-like structure close to the star. They also showed that there is a correlation between Ca II luminosity and IR excess in these stars and concluded that Ca II emission lines are somehow related to the presence of a disk.

Böhm \& Catala (1995) studied non-photospheric lines such as the $\mathrm{H} \alpha$, the Ca II IR triplet, and He I $(\lambda 5876)$. They concluded that the energy fluxes for these lines increase with the effective temperature and suggest that the origin of the emission in the lines is further away from the layer between the disk and the stellar surface. Heŕnandez et al. (2004) found that $33 \%$ of their sample showed emission in Fe II multiplets and also noticed that the equivalent width (EW, hereafter) of one of the Fe II lines, Fe II multiplet (42) ( $\lambda 5169)$, is correlated with the equivalent width of $\mathrm{H} \alpha$ and seems to be present only when [O I] $(\lambda 6300)$ also appear in the spectrum.

\subsection{Overview}

Differences in polarization, magnetism, and spectral lines between HAe and HBe stars suggest the possibility of a different accretion mechanisms at work. The accretion mechanism may be constrained by studying material very close to the star, determining its structure and kinematics. As high temperatures are expected close to the star, we chose emission lines that form outside the stellar photosphere and require high temperatures to be excited. Modeling these lines and investigating what kind of disk density structure they require will allow us to qualitatively understand the regions close to the star.

In this work, we model emission lines thought to be produced in the inner gaseous disk of one $\mathrm{HBe}$ star, BD+65 1637, using observations from CFHT 
ESPaDOnS. Section 3 describes the observations, reduction methods, and the emission lines found in the spectrum. The details of the models can be found in Section 4 and results of the modeling for each emission line can be found in Section 5 . The uniqueness of the models is discussed in Section 6. The paper concludes with a discussion in Section 7, and a summary of key findings in Section 8

\section{3. $\mathrm{BD}+651637$}

$\mathrm{BD}+651637\left(\mathrm{~V}^{*} \mathrm{~V} 361 \mathrm{Cep}\right)$ is a B2e star of visual magnitude 10.83 in the young cluster NGC 7129 (Straižys et al. 2013: Dahm \& Hillenbrand 2015). BD+65 1637 was identified as HBe star by George Herbig in his first paper on Herbig stars (Herbig 1960) and noted to a have spectrum very much like that of a Classical Be star. Hillenbrand et al. (1992) studied the star's spectral energy distribution (SED) which showed a small infrared excess, and they classified it as very similar to Classical Be stars. The assigned spectral type has varied in the literature from B2 to B5 (see Herbig (1960); Strom et al. (1972); Finkenzeller \& Mundt (1984); Finkenzeller (1984); Hillenbrand (1995)). Here, we will adopt the stellar parameters and their uncertainties from Alecian et al. (2013), reproduced in Table 1

Table 1:: Stellar parameters for BD+65 1637

\begin{tabular}{ll}
\hline \hline Parameter & Value \\
\hline Spectral Type & B2e \\
$\mathrm{T}_{\text {eff }}(\mathrm{K})$ & $18000 \pm 1000$ \\
$\log g(\mathrm{cgs})$ & 4.0 \\
Radius $\left(\mathrm{R}_{\odot}\right)$ & $6.7 \pm 0.7$ \\
Mass $\left(\mathrm{M}_{\odot}\right)$ & $8.11_{-0.23}^{+0.24}$ \\
Distance $(\mathrm{pc})$ & $1250 \pm 50$ \\
$v \operatorname{sini}\left(\mathrm{km} \mathrm{s}^{-1}\right)$ & $278 \pm 27$ \\
\hline
\end{tabular}

All the values for the parameters as well as their uncertainties are taken from Alecian et al. (2013).

\subsection{Observations}

The observational data were obtained in 2006 (HJD-2453898) using the high-resolution spectropolarimeter ESPaDOnS at the Canada-France-
Hawaii Telescope. Additional spectra are also available from the Narval spectropolarimeter at the Télescope Bernard Lyot, obtained in 2009 (HJD-2455099). Both spectropolarimeters cover the wavelength range from 3700 to $10500 \AA$ with a spectral resolution of 65,000 . The peak SNR per CCD pixel at $7300 \AA$ was 237 for ESPaDOnS spectrum and 276 for Narval spectrum.

BD+65 1637's CFHT spectrum can be seen in Figure 2 The spectrum not only contains strong Balmer line emission, $\mathrm{H} \alpha$ and $\mathrm{H} \beta$, but also emission in many metal lines such as those from calcium, oxygen and iron. In addition to the two strong Balmer lines, we will investigate one of the three Ca II infrared triplet lines $(\lambda 8542)$, two Fe II lines ( $\lambda 5169$ of multiplet 42 and $\lambda 5317$ of multiplet 49$)$ and one He I line $(\lambda 6678)$. The He I line is seen in absorption and is used to estimate stellar properties such as the $v$ sini of the star. A detailed profile of each line can be found in Figure 3.

The Balmer lines, $\mathrm{H} \alpha$ and $\mathrm{H} \beta$, are the strongest emission lines in the optical/NIR spectrum of $\mathrm{BD}+651637$, as illustrated in Figure 2. For $\mathrm{BD}+651637$, the equivalent width of the $\mathrm{H} \alpha$ lines has been noted to vary from $-45 \AA$ to $28 \AA$ (Heŕnandez et al. (2004), Fernández et al. (1995), Finkenzeller \& Mundt (1984) and Garrison \& Anderson (1977)). In the $2006 \mathrm{CFHT}$ spectrum, the EW measured for $\mathrm{H} \alpha$ is $-26.2 \AA$ and for $\mathrm{H} \beta,-0.92 \AA$. The EW for Ca II IR Triplet $(\lambda 8542)$ is measured to be $-4.0 \AA$. The two Fe II lines, $\lambda 5169$ and $\lambda 5317$, were chosen for this study as they are in different multiplets, and their EWs were measured to be $-0.58 \AA$ and $-0.40 \AA$. Fe II $\lambda 5169$ is in multiplet $42\left(a^{6} S-z^{6} P^{\circ}\right)$ and Fe II $\lambda 5317$ is in multiplet $49\left(a^{4} G-z^{4} F^{\circ}\right)$, although it is blended with a weaker line from multiplet 484 Both of these lines have low excitation energy. He I $(\lambda 6678)$ is in absorption, and the EW was measured to be $0.33 \AA$.

A comparison between the CFHT and Narval spectra, taken approximately three years apart, can be seen in Figure 3. Some variation in the spectral lines is seen, most notably in the metal lines and $\mathrm{V} / \mathrm{R}$ ratio, with a strong red component in Narval (2009) observations. The similar-

\footnotetext{
${ }^{4}$ While both multiplets arise from the $a^{4} G$ lower level, the $\Delta_{j i}$ value of the multiplet 48 component is 6.5 times smaller than the multiplet 49 component.
} 

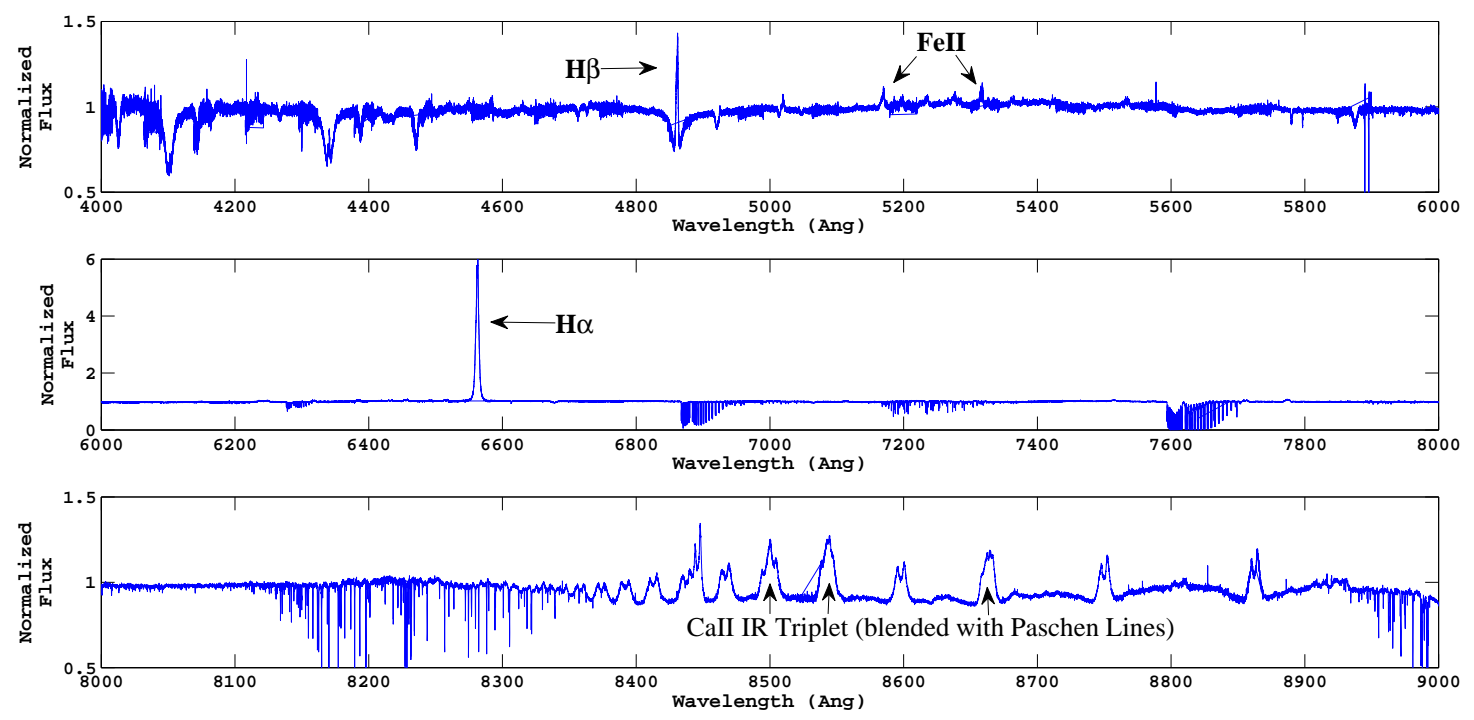

Fig. 2.- The observed spectrum for BD+65 1637 (Alecian et al. 2013). The spectrum shows many emission lines, such as the hydrogen Balmer lines, $\mathrm{H} \alpha(\lambda 6563)$ and $\mathrm{H} \beta(\lambda 4861)$, the Ca II IR-triplet lines $(\lambda 8498$, $\lambda 8542$ and $\lambda 8662$ ) and the Fe II lines ( $\lambda 5169$ of multiplet 42 and $\lambda 5317$ of multiplet 49 ). The strength of the lines vary over a large scale, particularly for $\mathrm{H} \alpha$ as seen in the vertical scale of each subplot.

ity in the He I ( $\lambda$ 6678) absorption line, in contrast to variations in the Balmer and metal lines, confirms that the Balmer and metal emission lines arise from material outside the star and that the disk structure varies over time. For the analysis of this paper, only the 2006 CFHT ESPaDOnS data was used.

$\mathrm{H} \alpha, \mathrm{H} \beta$, and both the FeII lines ( $\lambda 5169$ and $\lambda 5317$ ) show double-peaked profiles, with a stronger red component (R) when compared to the blue component $(\mathrm{V})$. All the non-photospheric lines included in this study show changes in the $\mathrm{V} / \mathrm{R}$ ratio over time (see Figure 3). Classical Be stars are known to show $\mathrm{V} / \mathrm{R}$ line variability which is interpreted as caused by a one-armed, global disk oscillation (for details see the review by Rivinius et al. (2013)). This effect has not been included in the model here, and hence the synthetic line profiles cannot fit both peaks of a line profile simultaneously.

\subsection{Reduction of Spectra}

In order to compare observed and modeled line profiles, the observed spectra needed to be contin- uum normalized. The unnormalized data obtained from CFHT (and Narval) were separated into specific wavelength windows that included the emission lines of interest. Each wavelength window was continuum normalized using IRAF. ${ }^{5}$ The function and order used for normalization varied from one spectral window to another; 'Legendre' and 'cubic spline' functions and low-order polynomials were generally used in the process.

The Ca II infrared triplet lines $(\lambda 8498, \lambda 8542$ and $\lambda 8662$ ) are blended with the hydrogen Paschen series. In order to compare with synthetic line profiles, the Paschen line must be subtracted from the Ca II line. This was done by taking an average of the two nearest, unblended Paschen lines and then subtracting the average from the Ca II line. Figure 4 illustrates all the three lines before the procedure of subtraction (on the left) and after the process of subtraction (on the right) in the CFHT spectrum. It can be seen that the

\footnotetext{
${ }^{5}$ IRAF is distributed by the National Optical Astronomy Observatories, which are operated by the Association of Universities for Research in Astronomy, Inc., under cooperative agreement with the National Science Foundation.
} 

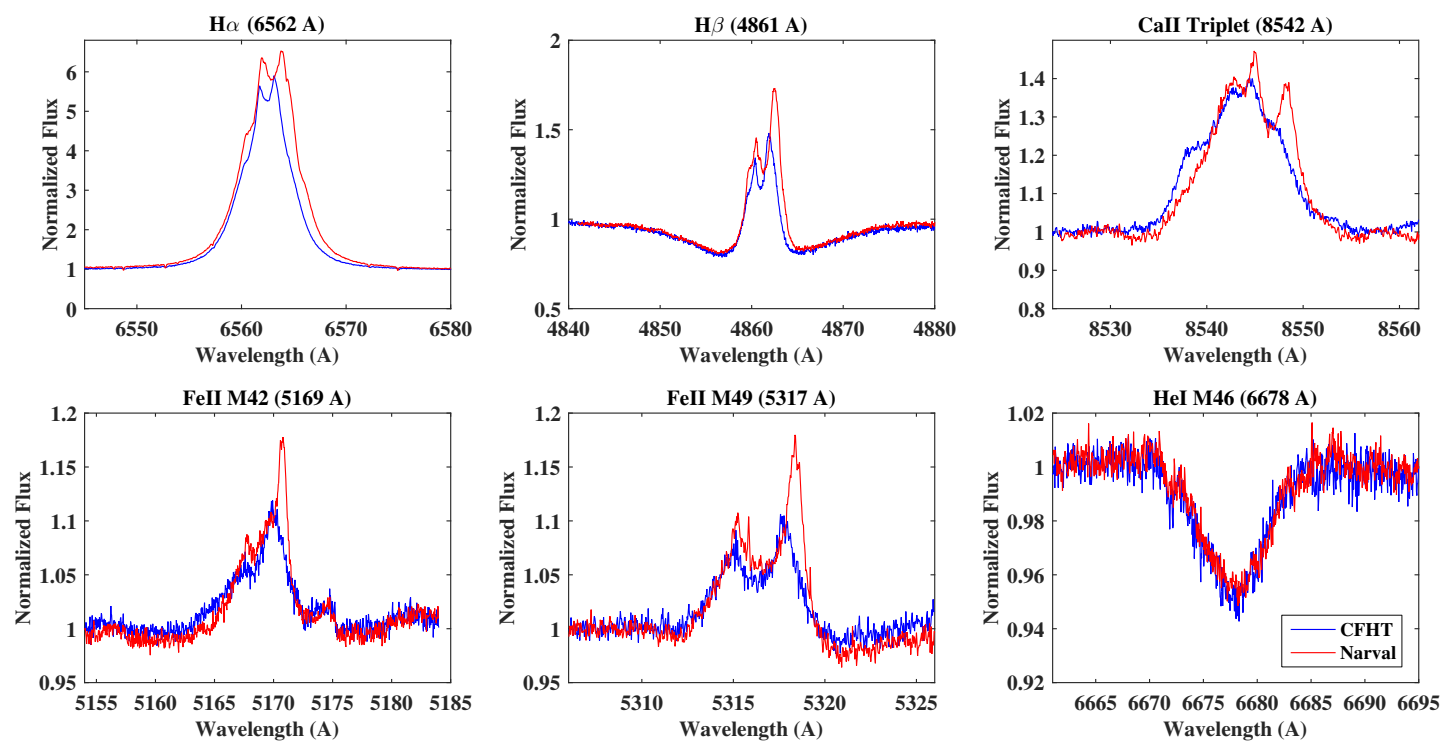

Fig. 3.- The CFHT ESPaDOnS observations (blue) are over-plotted with Narval observations (red) for each individual line. The ESPaDOnS observation was obtained in 2006 (HJD-2453898) at Canada-France-Hawaii Telescope, and the Narval observations were taken in 2009 (HJD-2455099) at the Télescope Bernard Lyot. Except for He I ( $\lambda 6678)$, all the lines show variations with time.

subtraction process decreases the strength of the Ca II line, and the resultant line profile is doublepeaked with a stronger red peak.

An example of the subtraction process is illustrated in Figure 5, where the average of Paschen $14(\mathrm{P} 14, \lambda 8596)$ and Paschen $17(\mathrm{P} 17, \lambda 8467)$ is subtracted from the blended Ca II $\lambda 8542$ line to extract the unblended Ca II profile. For the rest of the paper, the resultant Ca II line profile (seen in the right hand side panel of Figure 50 will be used. All the lines are adjusted for the stellar radial velocity. The radial velocity required for the line shifts was measured using the center of He I $(\lambda 6678)$, which was measured to be $-17.1 \mathrm{~km} \mathrm{~s}^{-1}$ for the CFHT spectrum and $-4.1 \mathrm{~km} \mathrm{~s}^{-1}$ for the Narval spectrum. Both the values are within the range of $-26 \pm 20 \mathrm{~km} \mathrm{~s}^{-1}$ measured by Alecian et al. (2013).

\section{Modeling}

To calculate the thermal structure of the equatorial, non-accreting, gaseous disk surrounding the central B star, the BEDIsK code (Sigut \& Jones 2007) was used. This code calculates the temper- ature structure of the disk, given a user-defined density structure, by enforcing radiative equilibrium in a gas of solar composition. The energy input into the disk was assumed to be solely from the photoionizing radiation field of the central star. The microscopic rates of heating and cooling were balanced to determine the temperature at many grid points in the disk. The disk was assumed to be axisymmetric and in Keplerian rotation about the central star ${ }^{6}$ BEDISK includes nine abundant elements (H, He, C, N, O, Mg, Si, Ca and Fe) over many ionization stages in the determination of the radiative equilibrium temperatures. The atomic level populations, required for the calculation of the heating and cooling rates, as well as for use later in computing emission lines, were obtained by solving the statistical equilibrium equations in an escape probability formalism (see Sigut \& Jones 【(2007) for details).

The user defined density structure of the disk $\left(\mathrm{g} \mathrm{cm}^{-3}\right)$ was taken to be specified by the parameters $\rho_{0}$ (base disk density) and $n$ (radial power

${ }^{6}$ No wind from the central star has been included in the calculations. 

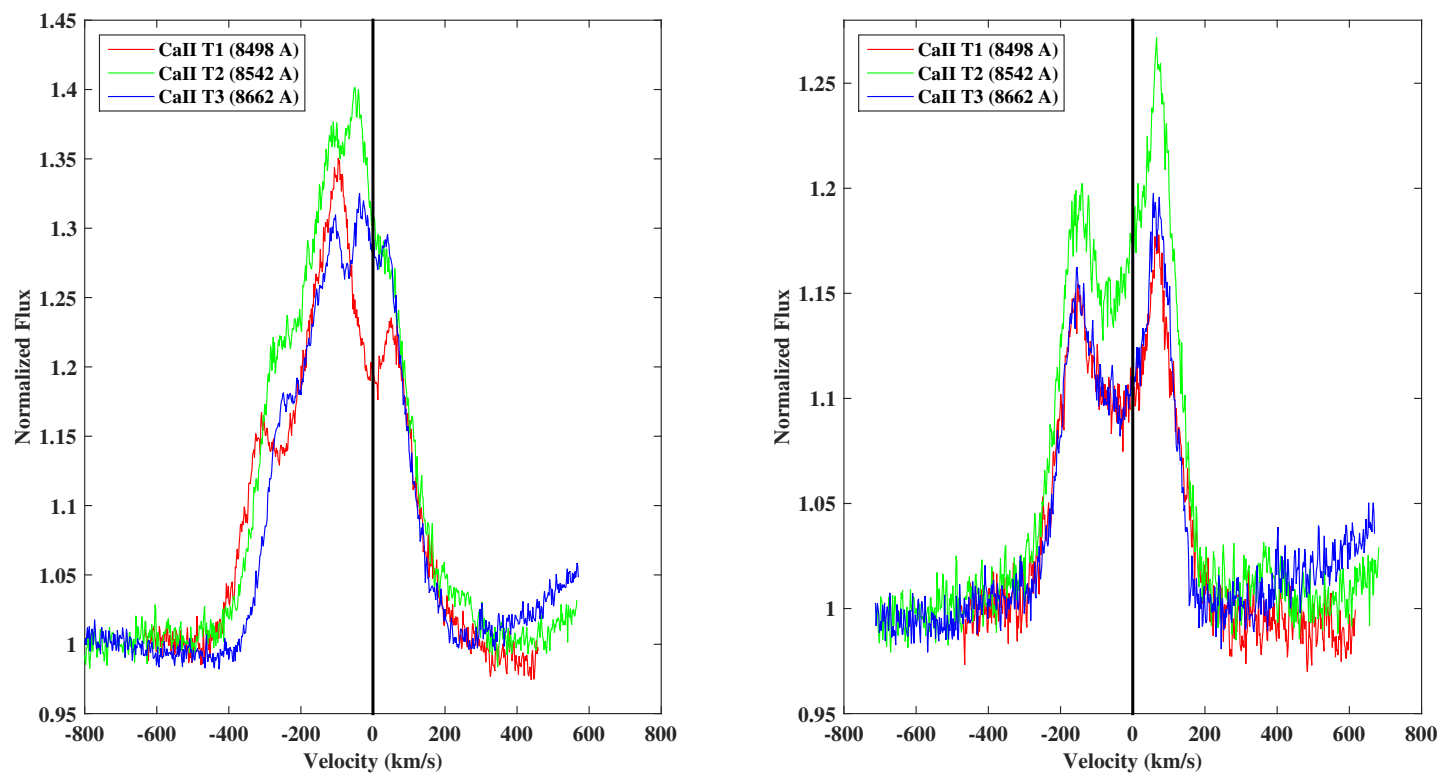

Fig. 4.- Left: All Ca II IR Triplets before the subtraction process (i.e. they are blended with Paschen lines). Right: The Ca II IR Triplets after subtraction of Paschen lines. The black solid line denotes $0 \mathrm{~km} / \mathrm{s}$ radial velocity.
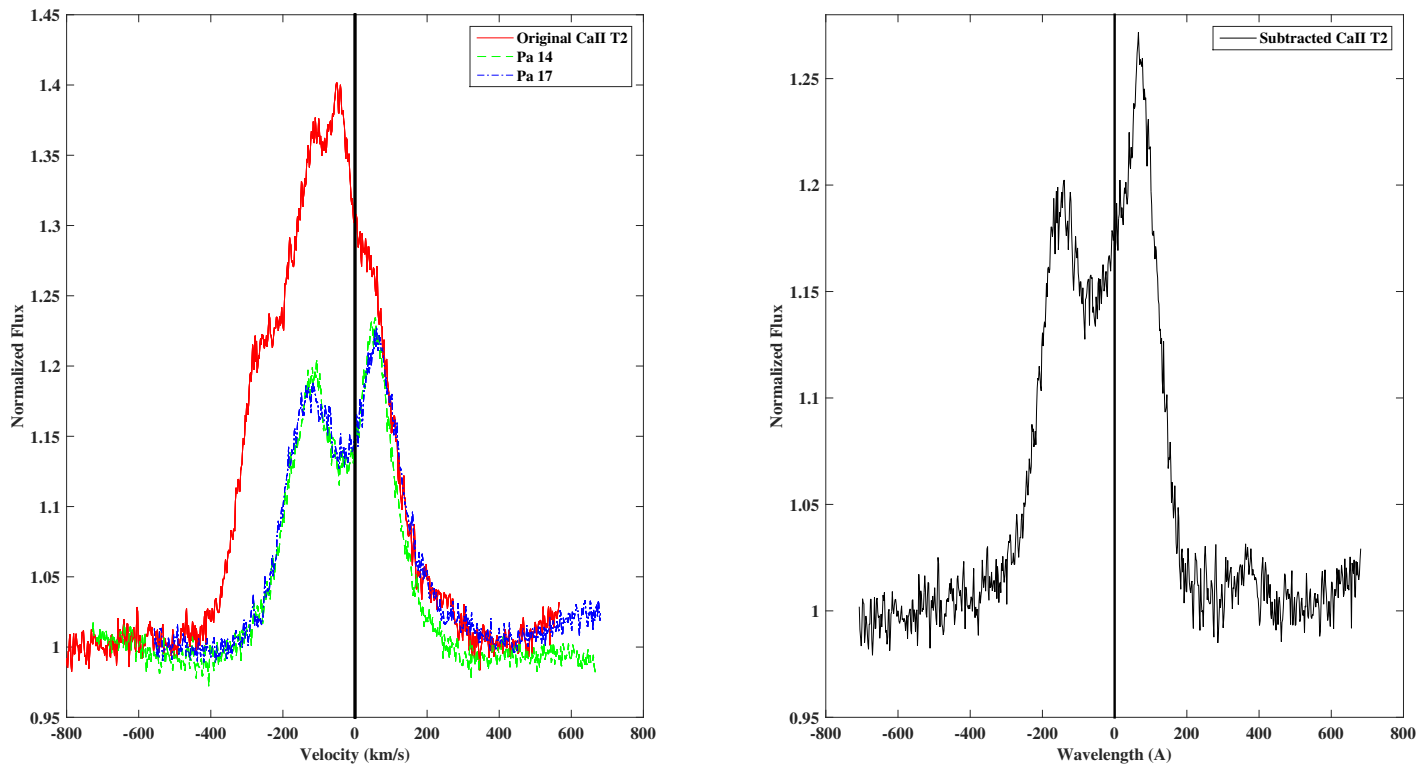

Fig. 5.- Left: One of the Ca II triplet lines $(\lambda 8542)$, seen in red, along with the Paschen $14(\mathrm{P} 14, \lambda 8596)$ in green and Paschen 17 (P17, $\lambda 8467)$ in blue. Right: The resultant Ca II line profile after subtraction of the average of the Paschen lines. The different scales on the y-axis should be noted. 
law index) in the equation

$$
\rho(R, Z)=\rho_{0}\left(\frac{R_{*}}{R}\right)^{n} e^{-\left(\frac{Z}{H}\right)^{2}},
$$

where $R$ and $Z$ are the cylindrical co-ordinates for the disk, $R_{*}$ is the stellar radius, and $H$ is the disk scale height. In all our models, the disk extends from the stellar photosphere ( $\mathrm{R}$ starts at $\mathrm{R}_{*}$ ) out to a radius of $\mathrm{R}_{\text {disk }}$.

If the vertical density structure of the disk is determined solely by gravitational equilibrium (the disk is rotationally supported in the $R$ direction), then the scale height has the form

$$
H=\beta\left(T_{\mathrm{HE}}\right)\left(\frac{R}{R_{*}}\right)^{3 / 2},
$$

where

$$
\beta\left(T_{\mathrm{HE}}\right)=\sqrt{\frac{2 k T_{\mathrm{HE}} R_{*}^{3}}{G M_{*} \mu M_{H}}} .
$$

Here, $M_{*}$ is the mass of the star, $\mu$ is the mean molecular weight of the gas in the disk (taken to be 0.68 ), and $T_{\mathrm{HE}}$ is the hydrostatic equilibrium temperature assumed for the disk. This hydrostatic temperature is used solely for setting the vertical scale height of the disk, and typically in Classical Be stars, one takes $T_{\mathrm{HE}} \approx 0.6 T_{\text {eff }}$. A self-consistent treatment, in which the radiative equilibrium disk temperatures are used in the calculation of the vertical hydrostatic equilibrium, is possible (see Sigut et al. (2009)), but that has not been used here. In the present work, varying $T_{\mathrm{HE}}$ allowed the scale height of the disk to be varied. Finally we note that vertical gravitational equilibrium predicts a flaring disk, $H \propto R^{3 / 2}$.

The assumption of vertical hydrostatic equilibrium produces a very thin disk near the star. From Eqn. 3. it is easy to see that the the ratio $H / R$ can be expressed as

$$
\frac{H}{R}=\frac{c_{S}}{V_{K}},
$$

where $c_{\mathrm{S}}$ is the local sound speed and $V_{\mathrm{K}}$ is the Keplerian orbital velocity. As the orbital velocity is typically hundreds of $\mathrm{km} \mathrm{s}^{-1}$ while the sound speed is on the order of $10 \mathrm{~km} \mathrm{~s}^{-1}$ for the disk temperature, the disk is predicted to be geometrically thin with $H / R \ll 1$. Such thin disks have been very successful in interpreting observables in Classical Be stars. ${ }^{7}$ but their application to HBe stars is less clear. For this reason, we also considered disk density models with an enhanced scale height, achieved by setting the $T_{\mathrm{HE}}$ temperature to be $5 T_{\text {eff }}$, a factor of nearly 10 over the gravitational equilibrium value. We will refer to these models as thick disk models. Note that for the same disk density parameters, $\rho_{0}, n$, and $R_{d i s k}$, the thick disk models are a factor of $\sqrt{5 / 0.6}$ more massive, as the total disk mass is proportional to the scale height.

Finally, we considered one additional modification to the basic disk model discussed above, although it is not an alteration to the density structure. In Classical Be stars, there is some evidence that the $\alpha$ viscosity parameter required in hydrodynamical models of disk formation and dissipation is $\approx 1$ (Carciofi et al. 2012). One possible interpretation of this result is that of sonic turbulence in the disk, i.e. $\nu=\alpha c_{S} H \sim c_{S} H$. For this reason, we have also considered models in which the disk is assumed to have a microtuburbulent velocity equal to the local sound speed. Microturbulence is a concept from classical stellar atmospheres that represents the dispersion of an assumed Gaussian distribution of turbulent velocities on scales smaller than unit optical depth. In this case, the turbulence acts to broaden the atomic absorption profile and hence is easily incorporated as an increase in the Doppler widths of radiative transitions. In our models, we assumed that the microturbulence value was either zero or equal to the local sound speed. These latter models will be referred to as turbulentdisks.

The temperature structure and atomic level populations computed by BEDISK are input into the code BERAY (Sigut 2011), which can compute observables such as line profiles, spectral energy distributions (hereafter SEDs) and monochromatic images in the sky. This is done by solving the equation of radiative transfer along a series of rays $\left(\approx 10^{5}\right)$ that pass through the star+disk system directed at the observer. Rays that terminate on the stellar surface use a Doppler-shifted photospheric (LTE) line profile for the initial boundary

\footnotetext{
${ }^{7}$ There has been some evidence in the support of larger disk scale heights (see Sigut \& Patel (2013); Arias et al. (2006); Zorec et al. (2007)).
} 
Table 2:: Explored model parameters for disk of BD+65 1637 .

\begin{tabular}{ll}
\hline \hline Parameter & Range \\
\hline Base Disk Density, $\rho_{0}\left(\mathrm{~g} \mathrm{~cm}^{-3}\right)$ & $10^{-8} \ldots 10^{-13}$ \\
Power Law Index, $n$ & $0.5 \ldots 3.0$ \\
Inclination, $i\left(^{\circ}\right)$ & $18 \ldots 75$ \\
Disk Radius, $R_{\text {disk }}\left(R_{*}\right)$ & $25 \ldots 100$ \\
\hline
\end{tabular}

condition, while rays that pass entirely through the disk assume no incident radiation. Note that the BERAY calculation adds an additional parameter, namely the viewing inclination of the system ( $i=0^{\circ}$ for a pole-on star face-on disk, and $i=90^{\circ}$ for an equator-on edge-on disk). Inclinations from $i=18^{\circ}$ to $i=75^{\circ}$ were computed. Finally, the computed spectral lines were convolved down to the instrumental resolution before comparing to the observed spectra.

\subsection{Classical Be Stars and the BERAY and Bedisk Codes}

BEDISK and BERAY are non-LTE radiative transfer codes constructed specifically for Classical Be stars and their ionized, gaseous decretion disks. Many studies, such as Silaj et al. (2014) and Silaj et al. (2010), have been able to successfully model the structure of the gaseous disk by comparing synthetic and observed $\mathrm{H} \alpha$ lines. Sigut 『2011) has shown that such models based on $\mathrm{H} \alpha$ are also able to correctly predict observed Fe II lines in the spectra of these stars. These models have also been able to reproduce the observed correlation seen between $\mathrm{H} \alpha$ and long term variations in visual magnitude, which are interpreted as formation and dissipation of the disk over a long periods of time in Classical Be stars (Sigut \& Patel 2013). Observed IR line fluxes (Jones et \begin{tabular}{l|l|l|l|l|}
\hline al. 2009: Halonen et al. 2008) as well as optical &
\end{tabular} and near-IR interferometry (Jones et al. 2008. Tycner et al. 2008: Mackay et al. 2009 Grzenia et al. 2013: Sigut et al. 2015) computed with BEDISK and BERAY models, have been used to put constraints on the several Classical Be star disks.

Several authors, such as Carciofi et al. 2009.
2007, 2006), studied the Classical Be stars $\alpha$ Eri, $\zeta$ Tau and $\delta$ Sco by fitting viscous decretion disk models to the observed Balmer lines, SEDs, and polarization measurements. Silaj et al. (2010) studied 56 Be stars and successfully fit the observed $\mathrm{H} \alpha$ profiles to BEDISK models. Many individual stars, such as $\chi$ Oph (Tycner et al. 2008), $\kappa$ Dra, $\beta$ Psc, $v$ Cyg (Jones et al. 2008), and $o$ Aqr (Sigut et al. 2015) have been studied spectroscopically as well as interferometrically, and are found to match a density model similar to the one adopted in this study. All of these mentioned studies, as well as several others, have fit the observed line profiles well with power law index $(n)$ ranging from 2 to 4 , typically 3.5 , and the disk base density varying between $10^{-10}$ and $10^{-12} \mathrm{~g} \mathrm{~cm}^{-3}$ (Rivinius et al. 2013).

Given the noted similarities between HBe and Classical Be stars (Hamann \& Persson 1992a

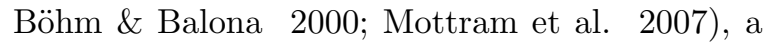
good starting point for the modeling of the emission spectra of HBe stars is using codes that have successfully been able to reproduce emission lines from the gaseous disks of Classical Be stars. The analysis can give insights on the regions where the lines are being formed, the mass of the disk, and the temperature and density structure of the emitting regions.

\section{Results}

Large libraries of synthetic line profiles were calculated for $\mathrm{H} \alpha, \mathrm{H} \beta$, the $\mathrm{Ca}$ II IR triplet $(\lambda 8542)$, Fe II $(\lambda 5169, \lambda 5317)^{8}$ and He I $(\lambda 6678)$ for disks surrounding a B2 star using combinations of the disk density parameters listed in Table 2. Values of the disk base density parameter, $\rho_{0}$, ranged from $10^{-13}$ to $10^{-8} \mathrm{gm} \mathrm{cm}^{-3}$, and the power-law index $n$ ranged from 0.5 to 3.0. Three different sized disks were considered, $\mathrm{R}_{\text {disk }}=25$, 50 and $100 \mathrm{R}_{*}$; thus, the largest disk considered has an outer diameter of $3.1 \mathrm{AU}$. This range of disk density parameters and disk sizes includes the range of values typically found for Classical Be stars, as noted above, but with an extension to more massive disks (i. e. higher $\rho_{0}$ and/or lower $n$ ). All synthetic line profiles were calculated at viewing inclinations of $18^{\circ}, 45^{\circ}, 60^{\circ}$ and $75^{\circ}$, which

${ }^{8}$ For Fe II 5317, only the multiplet 49 component was included. 
represent the centers of the first four bins of five equal-area bins in a random $\sin i$ distribution.

Each observed line profile was compared to its synthetic library by computing a figure-of-merit, $\mathcal{F}$, defined as

$$
\mathcal{F} \equiv \frac{1}{N} \sum_{i=1}^{N} \frac{\left|F_{i}^{\mathrm{Mod}}-F_{i}^{\mathrm{Obs}}\right|}{F_{i}^{\mathrm{Obs}}},
$$

where $F_{i}^{\mathrm{Obs}}$ is the observed relative flux, $F_{i}^{\mathrm{Mod}}$ is the model relative flux, and the sum is over the $N$ wavelength points spanning the line. In performing this sum, a range of small shifts to the observed wavelength scale was also tried, within the errors of the star's radial velocity. The smallest value of $\mathcal{F}$ was deemed to define the best-fit model for that feature, although all profiles with small values of $\mathcal{F}$ were visually inspected. In addition, the disk density parameters of profiles that fit the observed profiles almost as well as the bestfit model were also examined, and this point, concerning the uniqueness of the fits, will be discussed in Section 6 .

While the minimum of $\mathcal{F}$ for a given line, say $\mathcal{F}^{\mathrm{H} \alpha}$, defines the best fit for that particular line, it is not guaranteed that the best fit model for all lines will result in the same set of disk density parameters. A figure-of-merit defined by Eqn. 5 can be obtained for each line considered, i.e. $\mathcal{F}^{\mathrm{H} \alpha}$, $\mathcal{F}^{\mathrm{H} \beta}$, etc... Therefore, it is possible to search for the best set of disk parameters that minimizes the sum of all of the line figures-of-merit i.e. the global, best-fit model.

For all the line profile matches performed in this study, the effort was made to fit to the blue peak of the emission line. When a reasonable fit was not found for the blue peak, the fit was computed for the red peak instead.

We will now first discuss the best-fit models for each line individually, and then consider the best global model.

\subsection{Individual Fits}

The best-fit models for all individual lines are listed in Table 3 and the best synthetic line profile fits to the individual observed emission lines are shown in Figure 6. With the freedom to chose the density model independently for each line, the observed line profiles can be reproduced quite well in strength, shape, and equivalent width by the models.

The best fit for $\mathrm{H} \alpha$ is a $50 \mathrm{R}_{*}$, thin disk model with disk density parameter $\rho_{0}$ of $3.2 * 10^{-12} \mathrm{~g} \mathrm{~cm}^{-3}$ and power law index $n$ of 2.0 seen at at an inclination of $60^{\circ}$. The observed and synthetic profiles are compared in Figure 6. The width of $\mathrm{H} \alpha$ at its base is underestimated, and a better fit might be possible by refining the viewing inclination, however, we have not attempted this.

For $\mathrm{H} \beta$, the best match to the observed profile was found for a model with slightly smaller, thin disk of $25 \mathrm{R}_{*}$ seen at $45^{\circ}$ with disk density parameter $\rho_{0}$ of $1.0 * 10^{-11} \mathrm{~g} \mathrm{~cm}^{-3}$ and power law index $n$ of 2 . The overall strength and width of $\mathrm{H} \beta$ (including its absorption wings) are well reproduced by the model. We note that the $V / R$ asymmetry cannot be reproduced by our assumed axisymmetric disk models.

At this point, we immediately see that the bestfit models for $\mathrm{H} \alpha$ and $\mathrm{H} \beta$ differ. Nevertheless, it should be kept in mind that in addition to the best-fit model, there is a range of other disk models that fit each profile nearly as well. For example, there will be $\mathbf{N}$ models that fit $\mathrm{H} \alpha$ with a figure of merit within $25 \%$ of the best fit model, and for $\mathrm{H} \beta$, there will be $\mathbf{M}$ such models. We will return to the question of the number of such models and how the disk density parameter ranges compare in Section 6

For Ca II $\lambda 8542$, the best-fit model to the observed line has an disk density parameter $\rho_{0}$ of $1.0^{*} 10^{-10} \mathrm{~g} \mathrm{~cm}^{-3}$ and power law index $n$ of 2 seen at $60^{\circ}$ for a $25 \mathrm{R}_{*}$ thin and turbulent disk. We again note that the width and overall strength of the line are well reproduced.

For the two Fe II lines, the figure of merit $\mathcal{F}$ was computed by using only the red half of the line, i.e. the blue peak was ignored in the fit. The Fe II multiplet (42) $\lambda 5169$ line requires a disk density parameter $\rho_{0}$ of $1.0^{*} 10^{-10} \mathrm{~g} \mathrm{~cm}^{-3}$ and a power law index $n$ of 3 seen at $45^{\circ}$ for a $25 \mathrm{R}_{*}$ thick and turbulent disk. The Fe II multiplet (49) $\lambda 5317$ line requires a model with disk density parameter $\rho_{0}$ of $1.0^{*} 10^{-9} \mathrm{~g} \mathrm{~cm}^{-3}$ and a power law index $n$ of 1.5 seen at $75^{\circ}$ for a $25 \mathrm{R}_{*}$ thick and turbulent disk. We note that the lines of CaII and FeII prefer the turbulent disk model, as these models tend to produce broader and stronger lines. 
Table 3:: Best-fit model parameters for individual emission lines and the global models with and without Ca II.

\begin{tabular}{|c|c|c|c|c|c|}
\hline Emission Line & Disk Density $\rho_{0}\left(\mathrm{~g} \mathrm{~cm}^{-3}\right)$ & Power Law Index $n$ & Inclination $i\left(^{\circ}\right)$ & Disk Radius $R_{\text {disk }}\left(R_{*}\right)$ & Model Type \\
\hline $\mathrm{H} \alpha(6562 \AA)$ & $3.2 * 10^{-12}$ & 2.0 & 60 & 50 & Thin \\
\hline $\mathrm{H} \beta(4861 \AA)$ & $1.0 * 10^{-11}$ & 2.0 & 45 & 25 & Thin \\
\hline Ca II IR-triplet (8542 Å) & $1.0 * 10^{-10}$ & 2.0 & 60 & 25 & Thin \& Turbulent \\
\hline Fe II $(5169 \AA)$ & $1.0 * 10^{-10}$ & 3.0 & 45 & 25 & Thick \& Turbulent \\
\hline Fe II $(5317 \AA)$ & $1.0 * 10^{-9}$ & 1.5 & 75 & 25 & Thin \& Turbulent \\
\hline Global & $1.0 * 10^{-10}$ & 2.0 & 45 & 50 & Thin \\
\hline Global (w/o Ca II) & $1.0 * 10^{-10}$ & 3.0 & 45 & 50 & Thin \& Turbulent \\
\hline
\end{tabular}

Note.- No best-fit model for He I $(\lambda 6678)$ absorption line is given, as it is of photospheric origin.

To investigate what range of disk radii contribute to the formation of lines considered, the cumulative intensity produced by each emission line was plotted against the radius of the disk for the models listed in Table 3 as shown in Figure 7 To do this, a face-on synthetic image $\left(i=0^{\circ}\right)$ was produced using the best-fit disk density model for each line. For each $i=0^{\circ}$ image, the intensity was integrated over the total width of the line. Then, the integrated intensity out to a disk radius of $R$ can then be defined as

$$
C(R)=2 \pi \int_{R_{*}}^{R} I\left(R^{\prime}\right) R^{\prime} d R^{\prime}
$$

where $I(R)$ is the wavelength integrated line intensity at distance $R$, and $R_{*}$ is the stellar radius, assumed to be the inner edge of the disk. Then $C(R) / C\left(R_{\text {disk }}\right)$ can be plotted versus $R$ to determine how the line intensity is accumulated by the disk. In the Figure 7, a solid black line shows the cumulative fraction of 0.9 . It is important to keep in mind when looking at this figure that the disk density model particular to each transition has been used and not a single disk den- sity model. This explains, for example, why $\mathrm{C}=1$ is reached at $50 \mathrm{R}_{*}$ for $\mathrm{H} \alpha$, but $25 \mathrm{R}_{*}$ for the remaining lines. In order to reproduce the strength of the $\mathrm{H} \alpha$ emission, an extended emission region is required, reaching $90 \%$ of the emission at $40 \mathrm{R}_{*}$. However, $90 \%$ of the emission for Ca II, and Fe II $(\lambda 5169)$ originates from the inner most $10 R_{*}$ of the disk, and $\mathrm{H} \beta$ and Fe II ( $\lambda$ 5317) are intermediately reaching $90 \%$ complete at $\sim 20 \mathrm{R}_{*}$.

This figure also illustrates how the disk might be structured in order to produce all the line profiles by having the disk's equatorial density vary in a more general way than as a single power-law (see Section 7).

\subsection{Global Fits}

Given that different disk density models are required to best-fit each observed profile for $\mathrm{BD}+651637$, the next logical step was to see if a single disk density model could fit all the lines in a reasonable (as opposed to optimal) manner. This will also assist us in deciding how to move forward in looking for a more general density model that would better describe the structure of the 
disk. To find the single best model, we minimized the sum of all the individual $\mathcal{F}$, i.e.

$$
\mathcal{F}^{\text {total }}=\sum_{i=1}^{6} w_{i} F^{\mathrm{i}}
$$

where $i$ ranges over the six lines considered. Initially, we set $w_{i}=1$ for all $i$ to weigh all six lines equally. The model that was found to best reproduce all the observed line profiles in this manner is listed in Table 3 .

Figure 8 shows all six synthetic emission lines produced for this model as compared to the observed line profiles. The density parameters are a power law index $n$ of 2 , a disk density parameter $\rho_{0}$ of $1.0 * 10^{-10} \mathrm{~g} \mathrm{~cm}^{-3}$ with a $50 \mathrm{R}_{*}$, thin disk seen at $45^{\circ}$. As illustrated in the figure, the Balmer lines can be reproduced approximately in strength, but are too narrow at the base; the metal lines are either too strong (Fe II lines) or too weak (Ca II IR Triplet) compared to the observed emission lines. The mismatch of the shape of the Balmer line profiles, particularly in the wings, indicates that that the material is not distributed correctly in the disk by a single power-law. The synthetic line profile for Ca II IR triplet $\lambda 8542$ is weaker in strength, as well as narrower in velocity, than the observed line profile. It does, however, produce a double-peaked shape. Both of the synthetic Fe II line profiles have approximately the same shape and strength when compared to each other; however the width of the wings are different; Fe II (42) $\lambda 5169$ has narrower spread of velocity in the wings when compared to Fe II (49) $\lambda 5317$. Both synthetic Fe II line profiles are too strong compared to the observed profiles, suggesting that smaller regions may be required to reproduce the observed line (as seen in Section 5.1). Finally, He I shows absorption with some central emission, sometimes called a central quasi emission (CQE) feature (Hanuschik 1995). As the star and disk system is seen $45^{\circ}$, the CQE can be attributed to the disk which partly blocks the direct stellar radiation.

Figure 9 illustrates where the intensity is produced by these lines for this single power law model by plotting $\mathrm{C}(\mathrm{R})$, Equation 6 , as a function of disk radius. It can be seen that in all the cases, $90 \%$ of the emission now is coming from inside $40 \mathrm{R}_{*}$ (or $1.25 \mathrm{AU}$ ). This figure also illustrates that the CaII IR Triplet $(\lambda 8542)$ is produced in the innermost $10 \mathrm{R}_{*}$ of the disk; the $\mathrm{H} \beta(\lambda 4861)$ and the Fe II multiplets $(\lambda 5169 \& \lambda 5317)$ produce most of their emission within $20 \mathrm{R}_{*}$, and $\mathrm{H} \alpha$ emission is produced throughout the disk, with $90 \%$ coming from within $40 \mathrm{R}_{*}$. Comparison of Figures 7 and 9 shows that the Ca II IR triplet forms in the innermost part of the disk, while $\mathrm{H} \alpha$ forms throughout the disk. The emission from $\mathrm{H} \beta$ and Fe II multiplets are intermediate and emerge from the same region for the global model.

In addition to constraining the density distribution in the disk for a model that is consistent with the observations, the global model can be used to give insight to the temperature structure of the disk. Figure 10 illustrates the temperature distribution predicted by BEDISK in a thin disk for a model with a disk density parameter $\rho_{0}$ of $1.0 * 10^{-10} \mathrm{~g} \mathrm{~cm}^{-3}$ and power law index $n$ of 2 . The upper plot illustrates the temperature in the entire disk, which generally ranges from $5500 \mathrm{~K}$ to $10000 \mathrm{~K}$. The bottom log-log plot shows the region close to the stellar surface where temperatures in the disk can reach as high as $14000 \mathrm{~K}$. When combined with the density structure, this provides valuable information on structure of the inner, gaseous disk. For example, even at $110 R_{*}$, the coolest temperature predicted in the equatorial plane, $\simeq 5500 \mathrm{~K}$, is still above the dust sublimation temperature.

Finally, the disk density parameters can be used to estimate the total mass of the inner gaseous disk. The mass is estimated to be $9.3 * 10^{26} \mathrm{gm}$ $\left(5.7 * 10^{-8} M_{*}\right.$ or $\left.4.6 * 10^{-7} \mathrm{M}_{\odot}\right)$ while the scale height $H$ of the disk at the stellar surface was estimated to be $1.6 * 10^{10} \mathrm{~cm}\left(3.5 * 10^{-2} R_{*}\right.$ or 0.23 $\left.\mathrm{R}_{\odot}\right)$.

It was noticed in the fitting process that the metal lines, especially the Ca II IR Triplet, require a high disk density parameter $\rho_{0}$ with low value of the power law index $n$. CaII is generally not well reproduced by the models which otherwise are found to work reasonably well for a single power law. For this reason, we searched for global fits excluding the Ca II line by setting $w_{i}=0$ for $\mathcal{F}^{\text {Ca II }}$ in Equation 7. The result can be seen in Figure 11 The details for this model can be found in Table 3 and this model is able to reproduce the emission in the two Fe II lines reasonably well. The Balmer lines are not strong enough to match the strength of the observed profile. However they match well 
when the width of the lines is considered. The same is the case for He I line. The Ca II IR triplet for this model shows hardly any emission, and this indicates that Ca II is likely formed in a different region, while all the other lines can be reasonably produced by disk with a single power law with $n=3$. The mass of this disk was estimated to be $4.8 * 10^{25} \mathrm{gm}\left(2.3 * 10^{-9} M_{*}\right.$ or $\left.4.6 * 10^{-7} \mathrm{M}_{\odot}\right)$.

In order to illustrate where these lines are formed in this model which excludes CaII, and if there is any similarity to the previous global model, Figure 12 was constructed. The plot shows $\mathrm{H} \alpha$ forming almost throughout the entire disk with the $90 \%$ of emission coming from inside the $30 \mathrm{R}_{*}$. $\mathrm{H} \beta$, Fe II ( $\lambda$ 5169) and Fe II ( $\lambda$ 5317) can be seen forming within $15 \mathrm{R}_{*}$. When compared to the previous global fit model (Figure 9), all the emission lines except $\mathrm{H} \alpha$ in this model are produced within half the radius.

Thus, from the all three models considered, it can be concluded that the $\mathrm{H} \beta$ and the metal lines form in the innermost region of the disk while the $\mathrm{H} \alpha$ forms in an extended region covering nearly the entire disk.

\subsection{The Near-IR SED}

As mentioned in Section 4 Beray can also calculate continuum SEDs of the star+disk system. In order to assess how comparable these models are to the available observations, a SED was produced for the global disk model of Table 3 and compared to the observed SED for BD+65 1637 found in Hillenbrand et al. (1992). This is illustrated in Figure 13. The star's continuum SED, i.e. in the absence of a disk, is also shown. As it can be seen in the figure, the global disk model produces a brighter SED at longer wavelengths compared to the observed SED. This suggests a thinner and less dense disk than those considered here is required in order to be comparable to the observed SED. However, it is important to note that the SED observations were taken more than 16 years prior to the observations of the emission lines used in the analysis here. $\mathrm{H} \alpha$ has been previously reported to be variable in EW ( $-45 \AA$ to $-26 \AA$ ) and hence, the comparison of the disks over a long period of time should be considered with caution.

\section{Uniqueness of Disk Models}

As described in the Section 5, the fitting procedure used the values of the figure of merit $\mathcal{F}$ for all the lines, found by using Equation 7 , to build a set of the global, best-fit models. In Section 5.1 it was noted that although one model is the best-fit for each line profile, more than one model can fit a particular line profile within a certain range of $\mathcal{F}$. Table 4 gives the number of models for each emission line that have $\mathcal{F}<1.25 \mathcal{F}_{\text {min }}$ (i.e. the top $25 \%$ best fits). It can be seen from the table, the number of models within this range varies from 1 model for Ca II to 7 models for He I. Figure 14 illustrates where all these models fall in the explored parameter space of disk density $\rho_{0}$ and power law index $n$. If a single model is found, it is represented by a point. For two models, a line connecting the two models is shown on the figure. For three models, a triangle is used. For more than three models, an ellipse is shown that encloses most of the models. For HeI, a photospheric feature, the region that represents the models that reproduce no disk emission is shown with an arrow. It is important to keep in mind that this figure represents only the value of the power law index $n$ and disk density $\rho_{0}$; the rest of the parameters for the models $\left(R_{d i s k}\right.$ and $\left.i\right)$ are not distinguished. As the figure illustrates, some, but not all, of the models overlap, again illustrating that no common region is found where all of the lines can be well-fit by a single power-law model. However, two general regions on the plot can be separated, one for the Balmer lines, which require relatively low densities and another region of higher densities, dominated by the metal lines Fe II and Ca II. This figure confirms the earlier observation that the metal lines require higher densities.

Given that a single model is not able to reproduce the observed line profiles, understanding how different power law indices and disk densities for the disk affect the overall line strengths is important. To this end, the EW for each line as a function of disk density parameter $\rho_{0}$ was plotted for models with a $50 \mathrm{R}_{*}$ disk size seen at an inclination of $45^{\circ}$. Figures 15 and 16 show the results for the four disk types considered here: thin, thick, thin and turbulent, and thick and turbulent. Models with disk density parameter $\rho_{0}$ of $10^{-13}, 10^{-12}$, $10^{-11}, 10^{-10}$ and $10^{-9} \mathrm{~g} \mathrm{~cm}^{-3}$, and power law in- 
dices $n$ of 1,2 , and 3 are shown. In each figure, the black line indicates the observed EW for that particular emission line in the CFHT ESPaDOnS spectrum from 2006.

Even as no single $\left(\rho_{0}, \mathrm{n}\right)$ combination is able to match the observed EW of all the lines, some models match the observed EW for more than one line. For example, in Figure $15 \mathrm{~b}$ for a thin and turbulent disk, the EW of two Fe II lines and He I line match the observed EW for the disk density $\rho_{0}$ of $10^{-10} \mathrm{~g} \mathrm{~cm}^{-3}$ and power law index $n$ of 3 . The $\mathrm{EW}$ of $\mathrm{H} \alpha$ for the same model is very close to the observed EW. However, the EW of $\mathrm{H} \beta$ and Ca II $(\lambda$ 8542) are weaker for the same model when compared to the observations.

In general, the EWs increase with disk density $\rho_{0}$ to a maximum value, and then decline as the lines become saturated while the continuum continues to increase, weakening the EW. A good example of this can be seen for the power law index $n$ of 1 , where a sharp increase to a peak and than decline can be clearly seen for each line. In general, the maximum EW moves to a higher disk density $\rho_{0}$ as the power law index $n$ increases. Also illustrated in the plots, the addition of turbulence increases the strength of the lines. The thicker disk models generally show a large number of models with EW equal or greater than the observed EW for all the lines. For example, for a power law index $n$ of 2, the models with thicker disks show stronger EWs and a stronger rise in the EW as the density increases.

Table 4:: Number of models within $25 \%$ of the least value of the figure of merit $\mathcal{F}_{\text {min }} \leq \mathcal{F} \leq$ $1.25 \mathcal{F}_{\min }$ using the global disk density model of Table 3

\begin{tabular}{ll}
\hline \hline Emission Line & Number of Models \\
\hline $\mathrm{H} \alpha$ & 4 \\
$\mathrm{H} \beta$ & 7 \\
Ca II $(\lambda 8542)$ & 1 \\
Fe II $(\lambda 5169)$ & 3 \\
Fe II $(\lambda 5317)$ & 3 \\
\hline
\end{tabular}

\section{Discussion}

Good matches for all of the observed individual emission line profiles for BD+65 1637 have been found in the large library of synthetic models. However, the diversity of the models in Table 3 . and the failure to find one global model that fits all the observed line profiles well, seems to indicate that the density distribution within the inner gaseous disk of $\mathrm{BD}+651637$ cannot be of the simple form of a single power law (Equation 1) with power law index $n>0$. The differences between the best-fits for individual lines and global fits suggest that the structure of the disk is more complex than a single power law. The idea that different density structure might be at play is supported by Figure 7 which illustrates how the variations in the structure of the disk can produce all the emission lines. In addition, the metal emission lines (Ca II and Fe II) seem to require a denser region for their formation as compared to the Balmer lines.

The SED of the best-fit, global model overpredicts the near-IR excess compared to the available observations. However, it is important to note that SED is very sensitive to the underlying (assumed) stellar temperature in the optical/NIR, and hence should be viewed with caution. In general, the line modeling serves as a more powerful tool in inferring the structure of gaseous disk found close to the star. The comparison between the observed and computed SED was performed merely as a byproduct of the line modeling study performed here, and perhaps most importantly, the SED and line spectra observations are separated by 16 years.

A general trend was noticed while manually searching for the best-fit to the line profiles that the metal lines required higher densities compared to the Balmer lines in order to reproduce the observed line profiles. The addition of turbulence to these models made the lines stronger and broader.

Finally, the analysis of observed and synthetic line profiles and their fits suggest that BD+65 1637 is seen at an angle between $45^{\circ}$ and $60^{\circ}$.

Decretion disks around Classical Be stars are generally modeled with a single power law for the density structure, as mentioned in Section 4 and Sigut \& Jones (2007). When hydrodynamic models are used, a more complex density structure is predicted (Carciofi 2011). As shown by the cur- 
rent work, HAeBe stars do not seem to follow a single power law for their disk structure, perhaps as expected. Thus, a disk with density described by several different power laws in different radial zones might be able to provide a better global fit to all the lines considered. Finally, we assumed that the disk extends all the way to stellar photosphere, so another possible area of exploration would be to have the disk start further away from star. If the star isn't actively accreting, or has sporadic events of accretion, the disk may not extend all the way to the star. Many recent studies such as Vink (2015); Vink et al. (2005) have suggested that it may be possible to constrain the presence of such an inner hole radius using polarimetry.

\section{Conclusions}

This study of inner gaseous disk of the Herbig B2e star, BD+65 1637, by modeling the optical and near-infrared emission lines, has led to three key findings:

- All of the observed emission lines considered in this study can be reproduced with models that use photoionizing radiation of the central star as the sole energy source for the disk.

- Despite being able to reproduce the observed emission lines individually, no model based on a single power law for the equatorial density was able to reproduce all of the emission lines simultaneously. More complex density models are required to generate a consistent disk structure for this star.

- The metal lines (Ca II, Fe II) require higher densities when compared to the Balmer lines.

In addition to testing more general disk density models, and investigating the effect of an inner hole in the disk, we have three more B2-type stars and four B0-type stars in the database (Alecian et al. 2013). Applying the same modeling technique as in this work, we hope to further understand the overall geometry and structure of the disks around early-type HBe stars. For the next paper in this series, the B2-type stars, HD 76534, HD 216629 and HD 114981 will be analyzed.
Acknowledgments: This work is supported by the Canadian Natural Sciences and Engineering Research Council (NESRC) through Discovery Grants to T. A. A. Sigut and J. D. Landstreet.

\section{REFERENCES}

Alecian, E., Wade, G. A.; Catala, C., Folsom, C., Grunhut, J., Donati, J.-F., Petit, P., Bagnulo, S., Marsden, S. C., Ramirez Velez, J. C., Landstreet, J. D., Boehm, T., Bouret, J.-C. \& Silvester, J., 2008, CoSka, 38, 235A

Alecian, E., Wade, G. A., Catala, C., Grunhut, J. H., Landstreet, J. D., Bagnulo, S., Böhm, T., Folsom, C. P., Marsden, S. \& Waite, I., 2013, MNRAS, 429, 1001

Alecian, E., 2014, Putting A Stars into Context: Evolution, Environment, and Related Stars, Proceedings of the international conference held on June 3-7, 2013 at Moscow M.V. Lomonosov State University in Moscow, Russia. Eds.: G. Mathys, E. Griffin, O. Kochukhov, R. Monier, G. Wahlgren, Moscow: Publishing house "Pero", p. 84-92

Alonso-Albi, T.; Fuente, A.; Bachiller, R.; Neri, R.; Planesas, P.; Testi, L.; Berné, O. \& Joblin, C., 2009, A\&A, 497, 117A

Arias, M. L., Zorec, J., Cidale, L. \& Ringuelet, A. E., 2006, A\&A, 460, 821

Barklem, P. S. \& Piskunov, N., 2003, IAU Symposium 210, Modelling of Stellar Atmospheres, ed. N. Pisknouv, W. W. Wies \& D. F. Gray (San Francisco, CA: ASP), E28

Böhm, T. \& Balona, L. A., 2000, ASPC, 214, 103B

Böhm, T. \& Catala, C., 1995, A\&A, 301, 155B

Bouvier, J, Alencar, S. H. P., Harries, T. J., JohnsKrull, C. M. \& Romanova, M. M., 2007, Protostars and Planets V, 479B

Calvet N., Muzerolle J., Brice ${ }^{\sim n o}$ C., Hernández J., Hartmann L., Saucedo J. L. \& Gordon K. D., 2004, AJ, 128, 1294

Carciofi, A. C., Miroshnichenko, A. S., Kusakin, A. V., Bjorkman, J. E., Bjorkman, K. S., Marang, F., Kuratov, K. S., García-Lario, P.; Calderón, J. V. Perea; Fabregat, J.; Magalhães, A. M. 2007, ApJ, 2006, 652, 1617C 
Carciofi, A. C., Bjorkman, J. E., Miroshnichenko, A. S., Magalhães, A. M. \& Bjorkman, K. S., 2007, ASPC, 631, 242C

Carciofi, A. C., Okazaki, A. T., Le Bouquin, J. -B., Štefl, S., Rivinius, Th., Baade, D., Bjorkman, J. E. \& Hummel, C. A., 2009, A\&A, 504, 915C

Carciofi, A. C., 2011, IAUS, 272, 325C

Carciofi, A. C., Bjorkman, J. E., Otero, S. A., Okazaki, A. T., Štefl, S., Rivinius, T., Baade, D. \& Haubois, X., 2012, ApJ, 744L, 15C

Catala, C., 1994, ASPC, 62, 91C

Cauley, P. W. \& Johns-Krull, C. M., 2014, ApJ, $797,112 \mathrm{C}$

Cohen, M. \& Kuhi, L. V., 1979, ApJS, 41, 743C

Corcoran, M. \& Ray, T. P., 1997, A\&A, 321, 189C

Corcoran, M. \& Ray, T. P., 1997, A\&A, 331, 147C

Dahm, S. E. \& Hillenbrand, L. A., 2015, AJ, 149, 200D

Dullemond, C. P. \& Monnier, J. D., 2010, ARA\&A, 48, 205D

Eisner, J. A.; Lane, B. F.; Akeson, R. L.; Hillenbrand, L. A. \& Sargent, A. I., 2003, ApJ, 588, $360 \mathrm{E}$

Eisner, J. A., Lane, B. F., Hillenbrand, L. A., Akeson, R. L. \& Sargent, A. I., 2004, ApJ, 613, $1049 \mathrm{E}$

Fairlamb, J. R., Oudmaijer, R. D., Mendigutía, I., Ilee, J. D. \& van den Ancker, M. E., 2015, arXiv:1507.05967

Finkenzeller, U. \& Mundt, R., 1984, A\&AS, 55, 109.

Finkenzeller, U., 1985, A\&A, 151, 340

Finkenzeller, U. \& Jankovics, I., 1984, A\&AS, 57, $285 \mathrm{~F}$

Fernández, M., Ortiz, E., Eiroa, C. \& Miranda, L. F., 1995, ApSS, 114, 439

Garrison, L. M. \& Anderson, C. M., 1977, ApJ, 218,438
Gómez de Castro, A. I. 2013, Planets, Stars and Stellar Systems. Volume 4: Stellar Structure and Evolution, 279

Grzenia, B. J., Tycner, C., Jones, C. E., Rinehart, S. A., van Belle, G. T. \& Sigut, T. A. A., 2013, AJ, 145, 141G

Halonen, R. J., Jones, C. E., Sigut, T. A. A., Zavala, R. T., Tycner, C., Levine, S. E., Luginbuhl, C. B., Vlieg, N. \& Vrba, F. J., 2008, PASP, 120, 498H

Hamann, F. \& Persson, S. E., 1992a, ApJS, 82, $285 \mathrm{H}$

Hamann, F. \& Persson, S. E., 1992b, ApJS, 394, 628

Hanuschik, R. W., 1995, A\&A, 295, 423H

Herbig, G. H., 1960, ApJS, 4, 337H.

Hernández, J., Calvet, N., Briceño, C., Hartmann, L. \& Berlind, P., 2004, AJ, 127, 1682H

Hillenbrand, L. A., Strom, S. E., Vrba, F. J. \& Keene, J., 1992, ApJ, 397, 613

Hillenbrand, L. A. 1995, Ph.D. thesis, Univ. Massachusetts

Ilee, J. D., Wheelwright, H. E., Oudmaijer, R. D., de Wit, W. J., Maud, L. T., Hoare, M. G., Lumsden, S. L., Moore, T. J. T., Urquhart, J. S. \& Mottram, J. C., 2013, MNRAS, 429, 2960I

Ilee, J. D., Fairlamb, J., Oudmaijer, R. D., Mendigutía, I., van den Ancker, M. E., Kraus, S., \& Wheelwright, H. E., 2014, MNRAS, 445, $3723 \mathrm{I}$

Jain, S. K. \& Bhatt, H. C., 1995, A\&AS, 111, 399J

Jones, C. E., Tycner, C., Sigut, T. A. A., Benson, J. A. \& Hutter, D. J., 2008, ApJ, 687, 598J

Jones, C. E., Molak, A., Sigut, T. A. A., de Koter, A., Lenorzer, A. \& Popa, S. C., 2009, MNRAS, 392, 383J

Kraus, S., 2015, Ap\&SS, 357, 97K

Larson, R. B., 2003, RPPh, 66,1651L

Mackay, F. E., Elias, N. M., II, Jones, C. E., \& Sigut, T. A. A., 2009, ApJ, 704, 591M 
Mannings, V. \& Sargent, A., 1997, ApJ, 490, $792 \mathrm{M}$

Mannings, V. \& Sargent, A., 2000, ApJ, 529, $391 \mathrm{M}$

Mendigutía, I., Eiroa, C.; Montesinos, B., Mora, A., Oudmaijer, R. D., Merín, B. \& Meeus, G., 2011, A\&A, 529A, 34M

Millan-Gabet, R., Malbet, F., Akeson, R., Leinert, C., Monnier, J. \& Waters, R., 2007, PRPL.Conf, 539M (arXiv:astro-ph/0603554)

Monnier, J. D. \& Millan-Gabet, R., 2002, ApJ, $579,694 \mathrm{M}$

Monnier, J. D., Millan-Gabet, R., Billmeier, R., Akeson, R. L., Wallace, D., Berger, J.-P., Calvet, N., D'Alessio, P., Danchi, W. C., Hartmann, L., Hillenbrand, L. A., Kuchner, M., Rajagopal, J., Traub, W. A., Tuthill, P. G., Boden, A., Booth, A., Colavita, M., Gathright, J., Hrynevych, M., Le Mignant, D., Ligon, R., Neyman, C., Swain, M., Thompson, R., Vasisht, G., Wizinowich, P., Beichman, C., Beletic, J., Creech-Eakman, M., Koresko, C., Sargent, A., Shao, M. \& van Belle, G., 2005, ApJ, 624, 832M

Mottram, J. C., Vink, J. S., Oudmaijer, R. D. \& Patel, M., 2007, MNRAS, 377, 1363M

Perez, M. R. \& Grady, C. A., 1997, SSRv, 82, 407P

Rivinius, Thomas, Carciofi, Alex C. \& Martayan, Christophe, 2013, A\&ARv, 21, 69R

Silaj, J., Jones, C. E., Tycner, C., Sigut, T. A. A. \& Smith, A. D., 2010, ApJS, 187, 228S

Silaj, J., Jones, C. E., Sigut, T. A. A. \& Tycner, C., 2014, ApJ, 795, 82

Sigut, T. A. A. \& Jones, C. E., 2007, ApJ 668, 481.

Sigut, T.A.A., McGill, M. A., Jones, C.E., 2009, ApJ, 699, 1973-1981.

Sigut, T. A. A., 2011, in IAU Symposium 272: Active OB Stars: Structure, Evolution, Mass Loss, and Critical Limits, ed. C. Neiner, G. Wade, G. Meynet, \& G. Peters (Cambridge: Cambridge Univ. Press), 426.
Sigut, T. A. A \& Patel, P., 2013, ApJ, 765, 41S

Sigut, T. A. A., Tycner, C. T., Jansen, B. \& Zavala, R. T., 2015, ApJ, in press

Straižys, V. et al 2013, MNRAS, 000, 1-8

Strom, S. E., Strom, K. M., Yost, J., Carrasco, L., \& Grasdalen, G. 1972, ApJ, 173, 353

Tycner, C., Jones, C. E., Sigut, T. A. A., Schmitt, H. R., Benson, J. A., Hutter, D. J. \& Zavala, R. T., 2008, ApJ, 689, 461T

van den Ancker, M. E., de Winter, D. \& Thin A Djie, H. R. E., 1998, A\&A, 330, 145

Vink, J. S.; Drew, J. E.; Harries, T. J.; Oudmaijer, R. D., 2002, MNRAS, 337, 356V

Vink, J. S., Drew, J. E., Harries, T. J. \& Oudmaijer, R. D., 2005, ASPC, 343, 232V

Vink, J., 2015, Ap\&SS, 357, 98V

Waters, L. B. F. M. \& Waelkens, C., 1998, ARAA, 36,233

Wheelwright, H. E., Oudmaijer, R. D., de Wit, W. J., Hoare, M. G., Lumsden, S. L. \& Urquhart, J. S.

Zinnecker, H. \& Yorke, H. W., 2007, ARAA, 45, 481

Zorec, J., Arias, M. L., Cidale, L. \& Ringuelet, A. E., 2007, A\&A, 470, 239

This 2-column preprint was prepared with the AAS LATEX macros $\mathrm{v} 5.2$. 

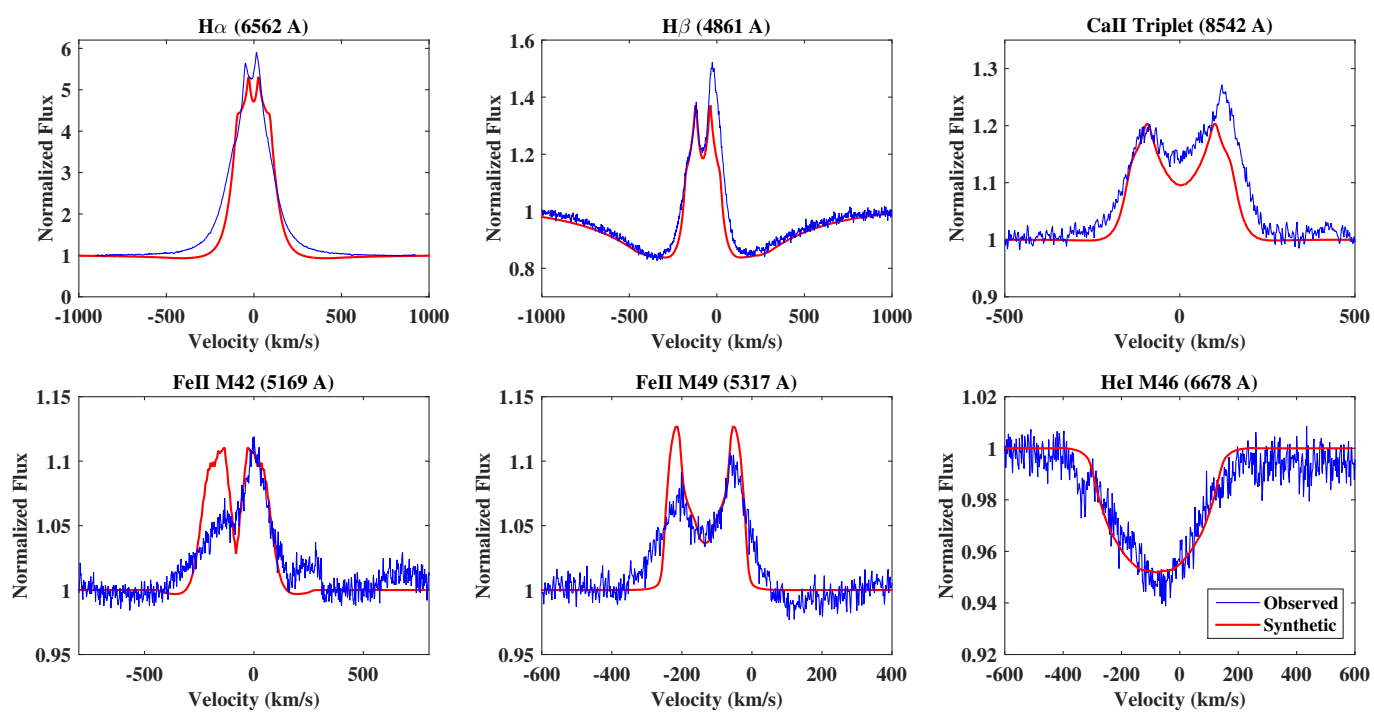

Fig. 6. - The best-fit for each line synthetic line profile (red) for the observed emission line (blue) of $\mathrm{H} \alpha$ ( $\lambda$ 6562), H $\beta$ ( $\lambda$ 4861), Ca II IR Triplet ( $\lambda$ 8542), Fe II multiplet (42) ( $\lambda$ 5169) and Fe II multiplet (49) $(\lambda$ 5317) and He I $(\lambda 6678)$ for BD+65 1637, which were modeled using BERAY. The fit parameters for each model can be found in Table 3 , with the exception of He I as it is fit by a photospheric profile.

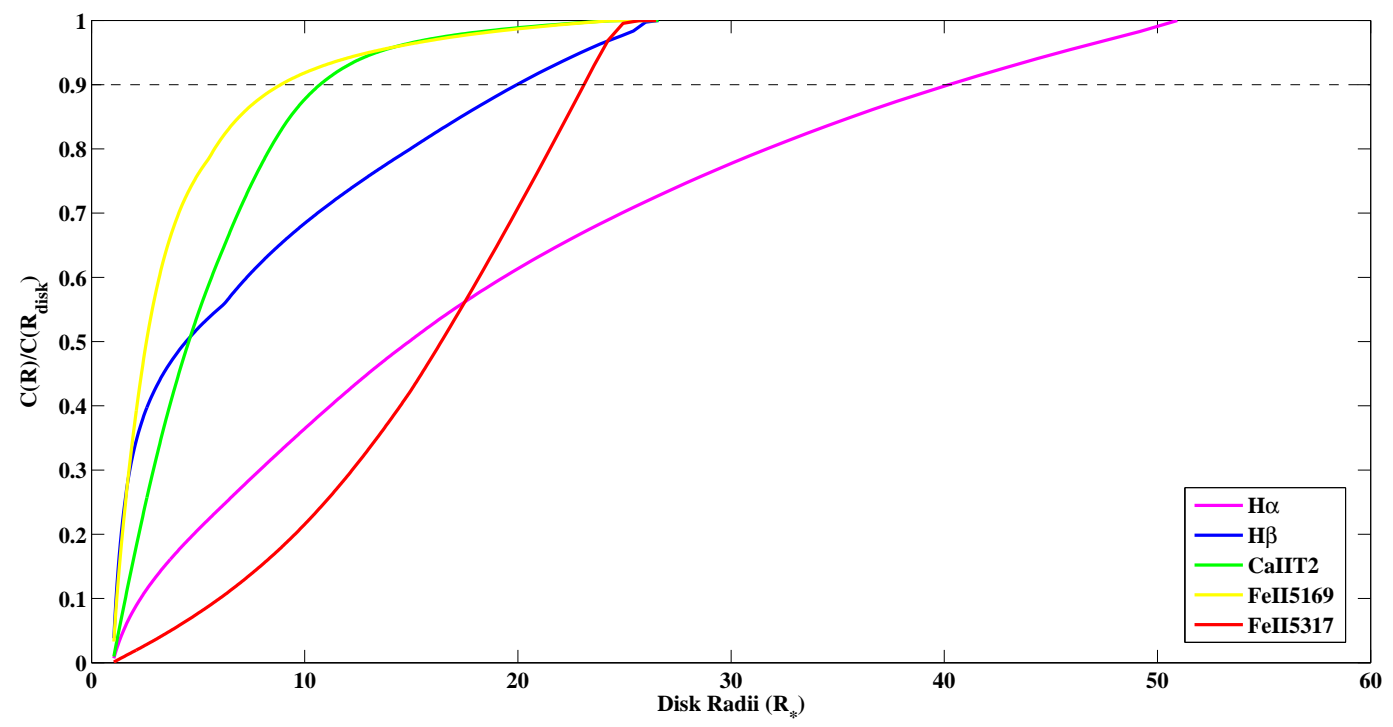

Fig. 7.- The cumulative line emission (Equation 6) as a function of distance from the star for each individual line using the best-fit disk density model. The model parameters that produced each individual line can be found in Table 3 . The black dashed line represents a cumulative emission fraction of 0.9. 

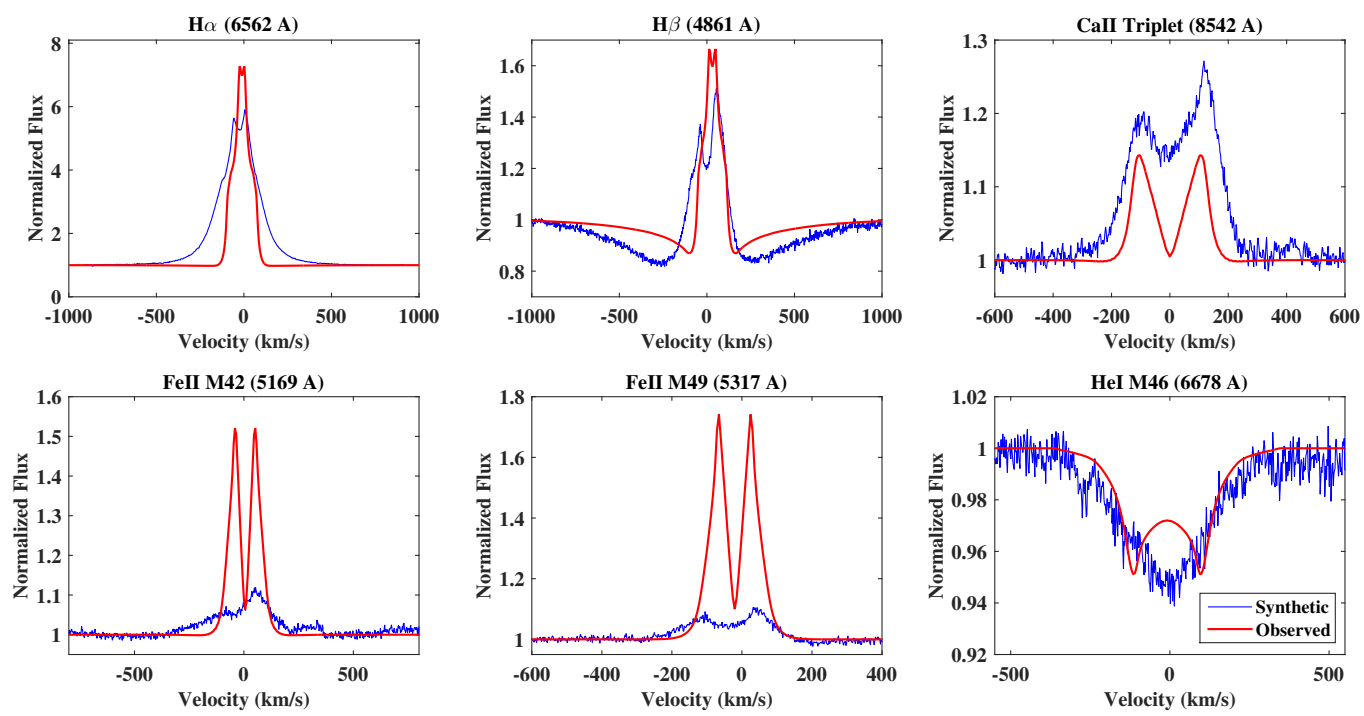

Fig. 8. - The global best-fit for all the synthetic line profiles (red) for the observed emission lines (blue) of $\mathrm{H} \alpha(\lambda 6562), \mathrm{H} \beta$ ( $\lambda$ 4861), Ca II IR Triplet ( $\lambda$ 8542), Fe II multiplet (42) ( $\lambda$ 5169) and Fe II multiplet (49) $(\lambda 5317)$ and He I $(\lambda 6678)$ for BD+65 1637, which were modeled using BERAY. The global best fit model parameters used for this Figure can be found in Table 3.

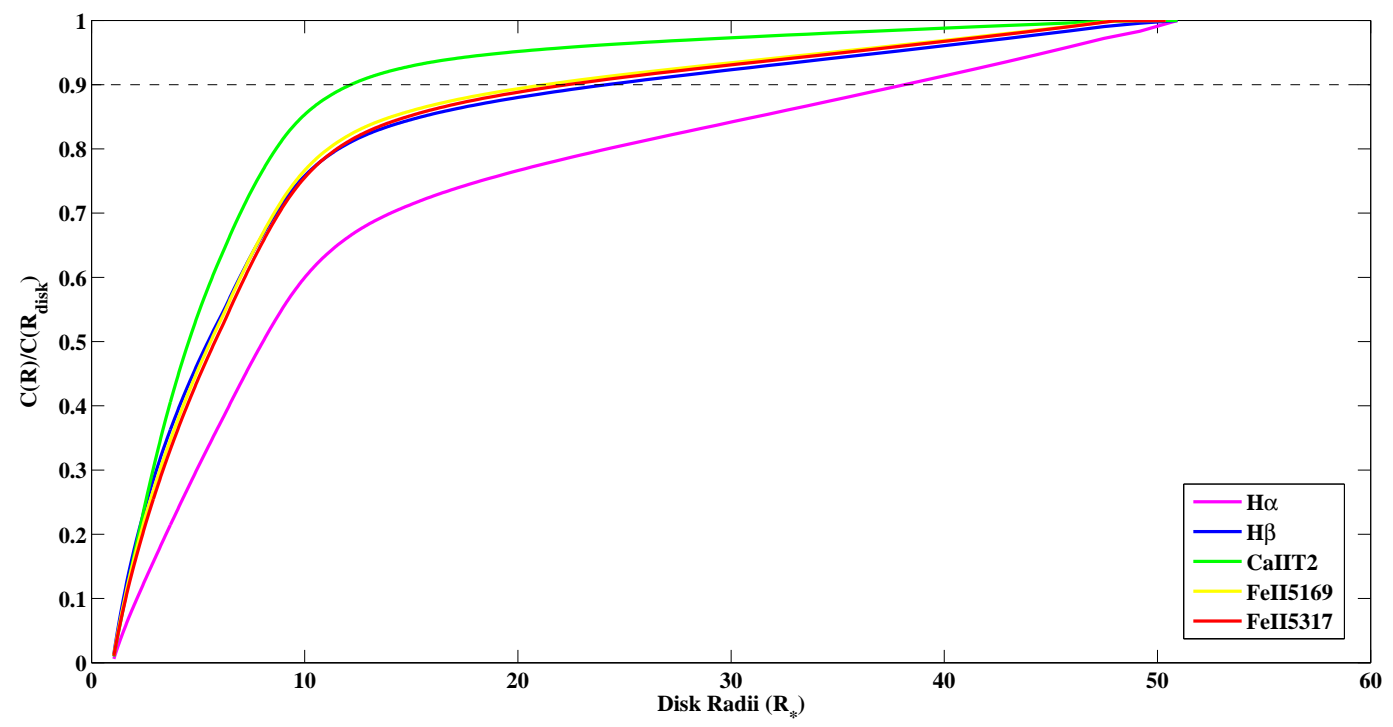

Fig. 9.- The cumulative line emission (Equation 6) as a function of distance from the star for each individual line using the global disk density model. The global model parameters can be found in Table 3 . The black dashed line represents from where the $90 \%$ of the emission is coming from. 

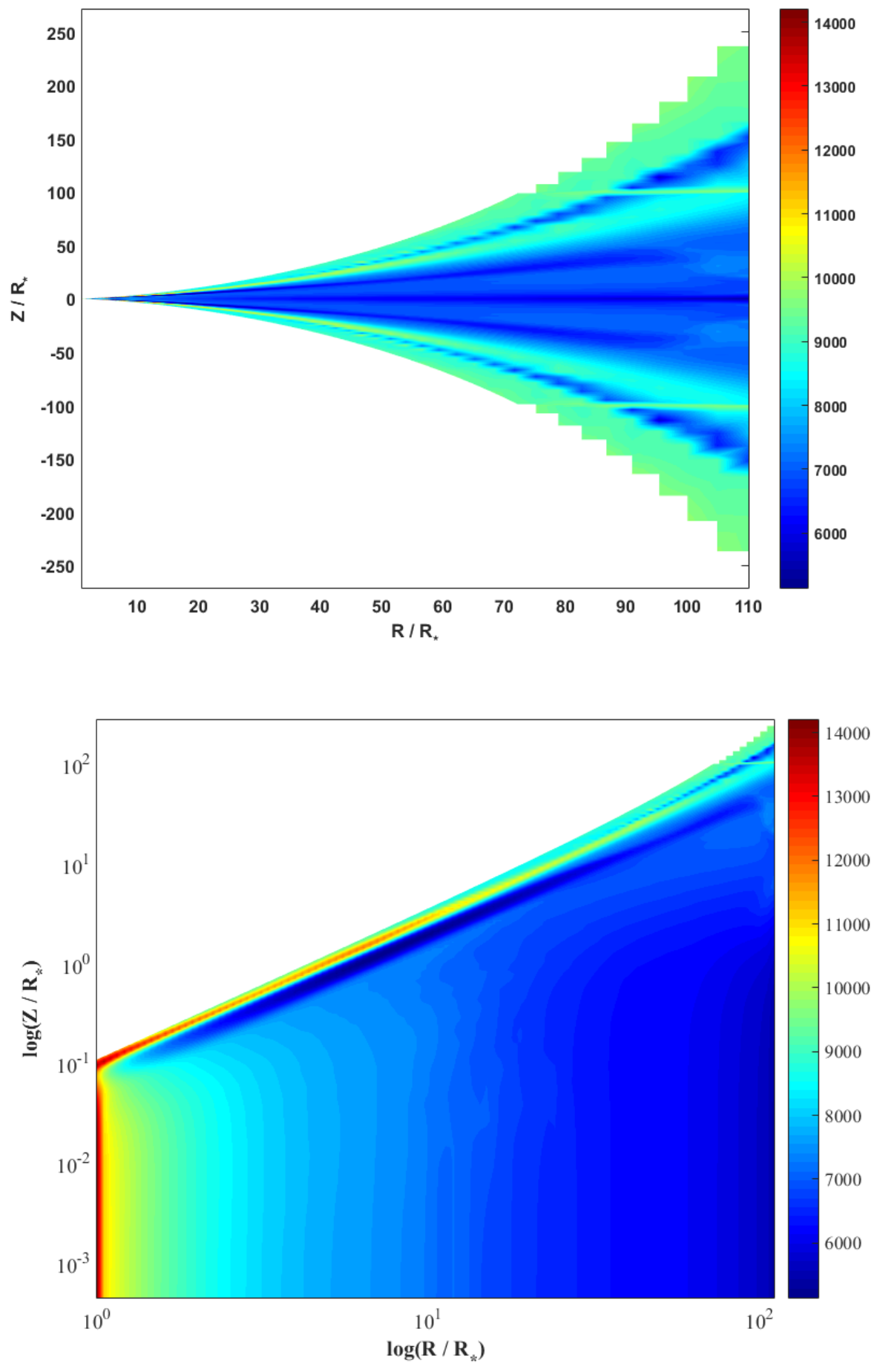

Fig. 10.- Temperature distribution in the disk for a model with disk density parameter $\rho_{0}$ of $1.0 *$ $10^{-10} \mathrm{~g} \mathrm{~cm}^{-3}$ and power law index $n$ of 2 . On the top, the plot illustrates the temperature structure in the entire disk, while at the bottom, in a log-log plot, the temperature distribution in the region close to the stellar surface is illustrated. A colorbar giving the temperature scale (in $\mathrm{K}$ ) is to the right of each plot. 

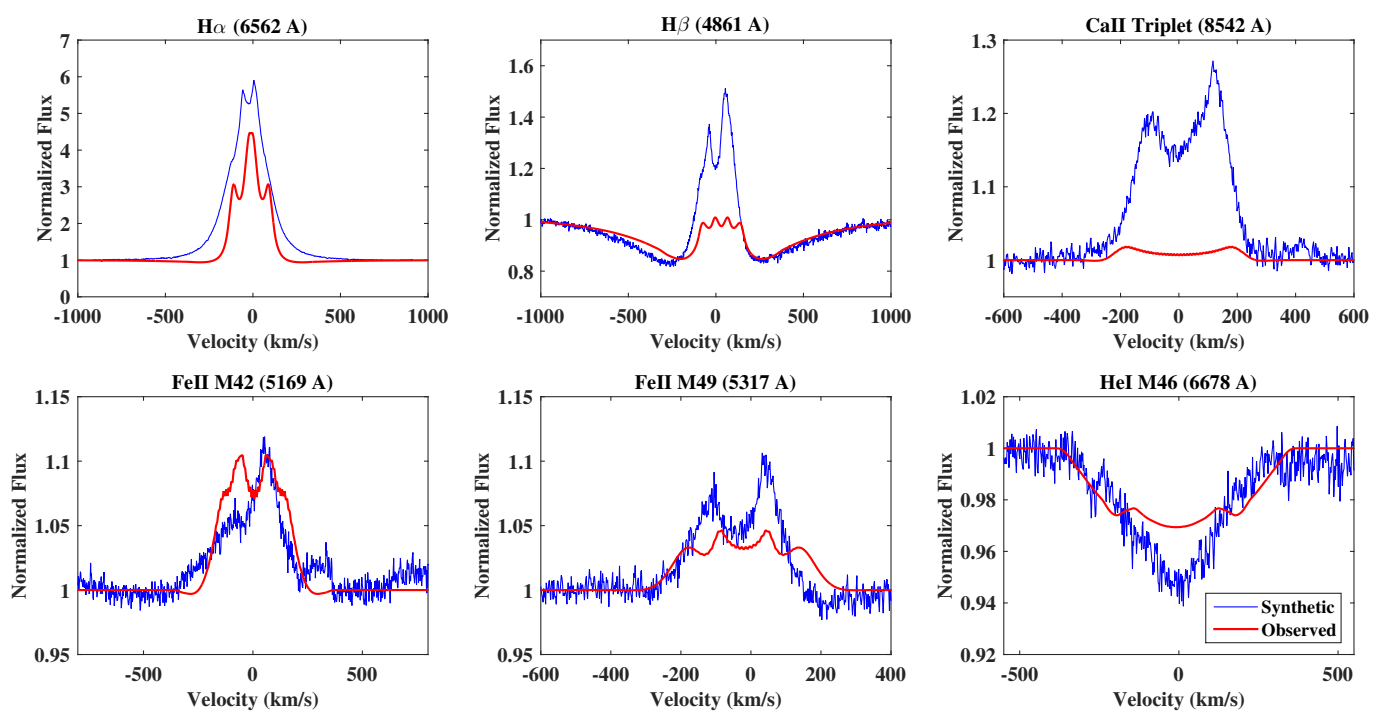

Fig. 11. - The global best-fit for the model that generates reasonable emission for all lines except Ca II IR Triplet $(\lambda 8542)$ when compared to the observed spectral lines (blue). The global model used can be found in Table 3 (on the line "w/o Ca II").

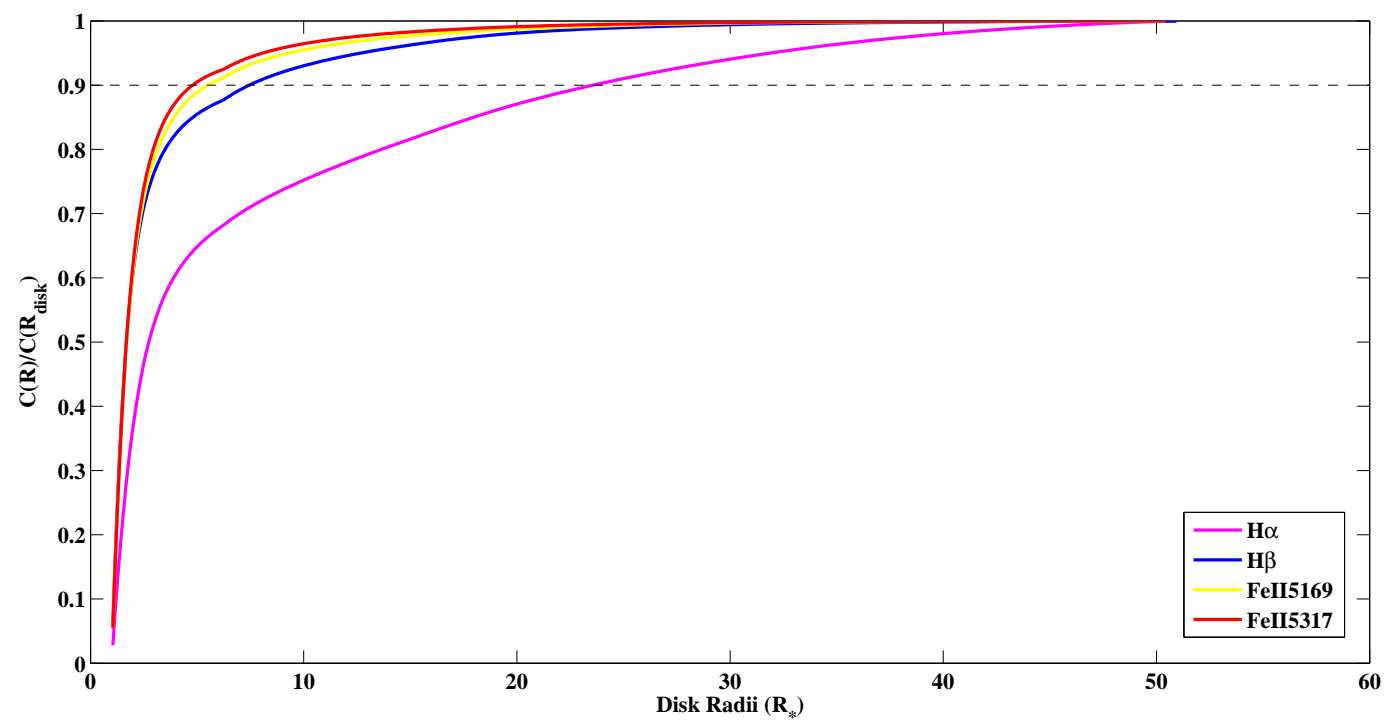

Fig. 12.- The cumulative line emission (Equation 6) as a function of distance from the star for each individual line using the global disk density model without Ca II. The model parameters used for this figure can be found in Table 3. The black dashed line represents from where the $90 \%$ of the emission is coming from. 


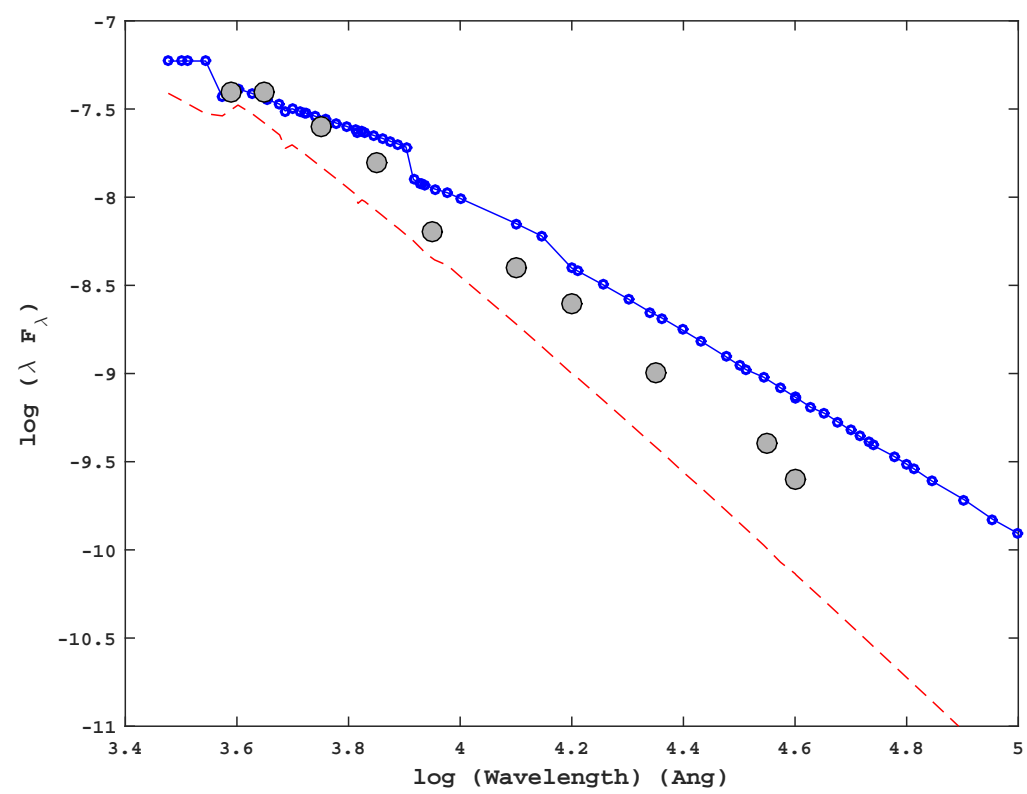

Fig. 13. - The continuum SED calculated using BERAY for the global best fit model (with Ca II) for the disk (blue circles). The photospheric spectrum of the star is shown as the red dashed line. These SED observations of Hillenbrand et al. (1992) are marked with gray filled circles. The global model used for this SED of the disk can be found in Table 3 .

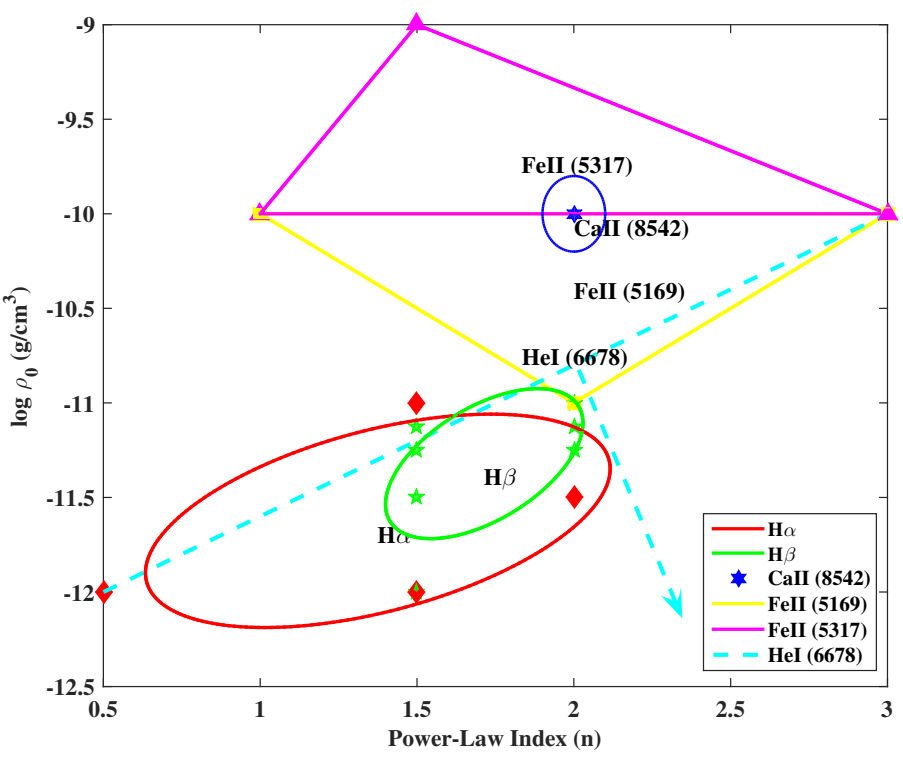

Fig. 14.- Regions of the (n, $\left.\log \rho_{0}\right)$ plane occupied by the top $25 \%$ of the profiles that match the observed profile individually (using the figure of merit values $\mathcal{F}) . \mathrm{H} \alpha(\lambda 6562)$ in shown in Red, $\mathrm{H} \beta(\lambda$ 4861) in Green, Ca II ( $\lambda$ 8542) in Blue, Fe II multiplet (42) ( $\lambda$ 5169) in Yellow and multiplet (49) ( $\lambda$ 5317) in Magenta, and He I $(\lambda 6678)$ in Cyan. the He I constraint is the region that results in no observable disk emission in the He I photospheric line. 

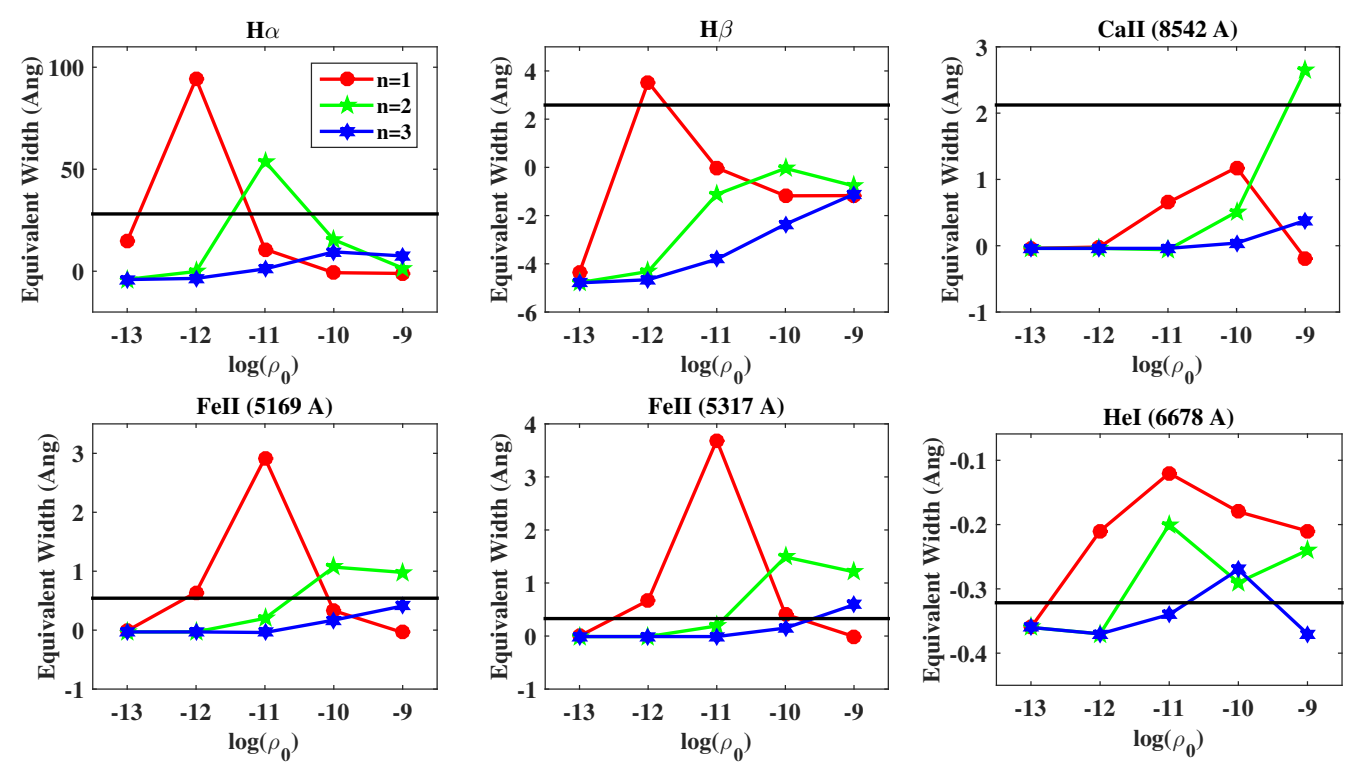

(a) Thin disk model
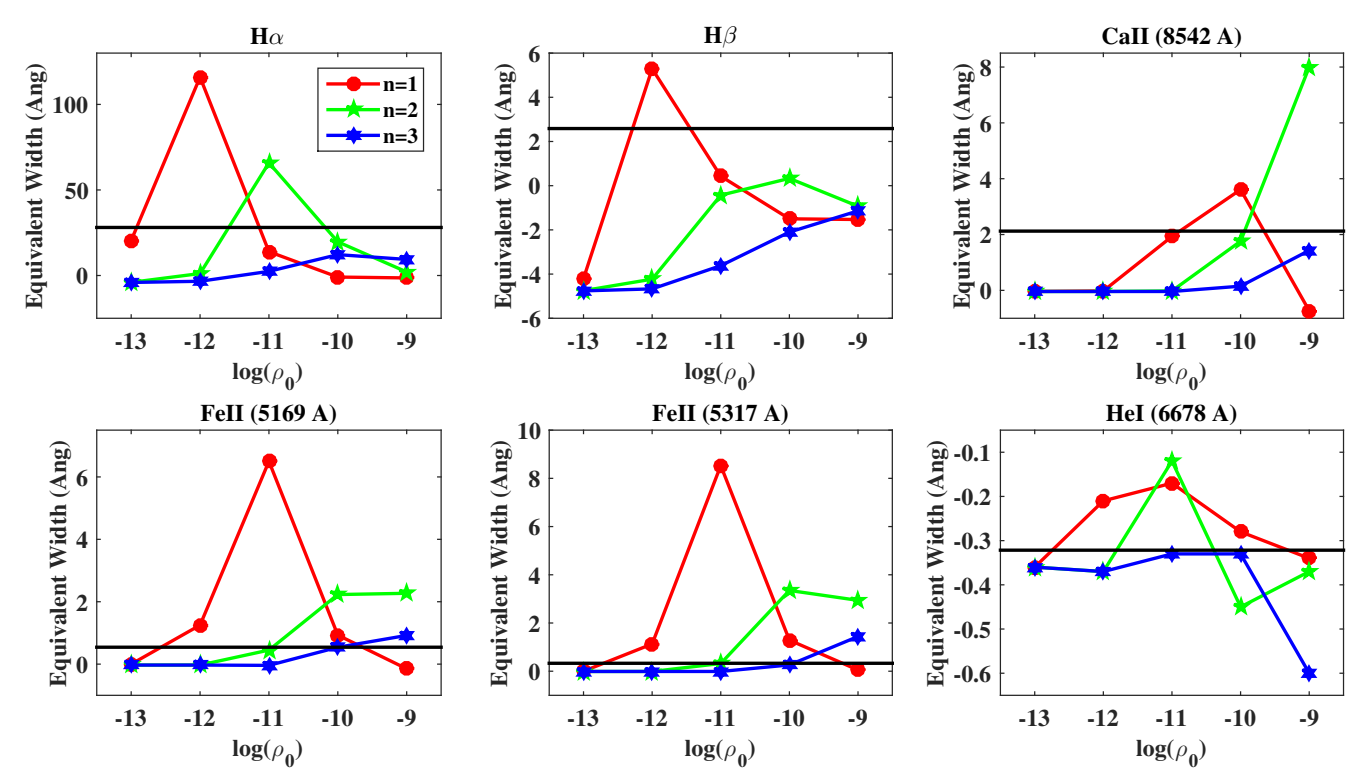

(b) Thin E Turbulent disk model

Fig. 15.- Equivalent width $(\AA)$ of each individual line as a function of density $\left(\log \left(\rho_{0}\right)\right)$ for three different power law indices $n$ for models of radius $50 \mathrm{R}_{*}$ seen at $45^{\circ}$ inclination angle. The solid black line in each panel indicates the respective observed equivalent width. 

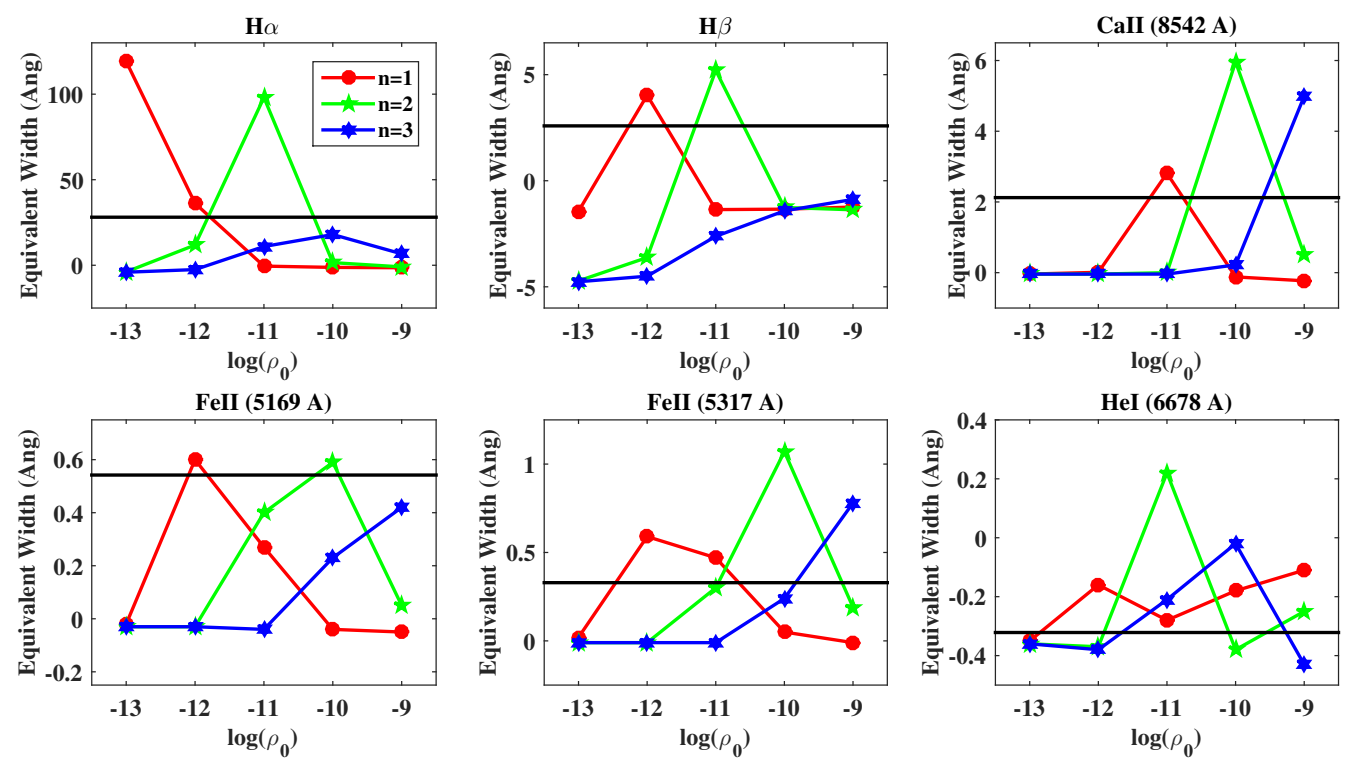

(a) Thick disk model
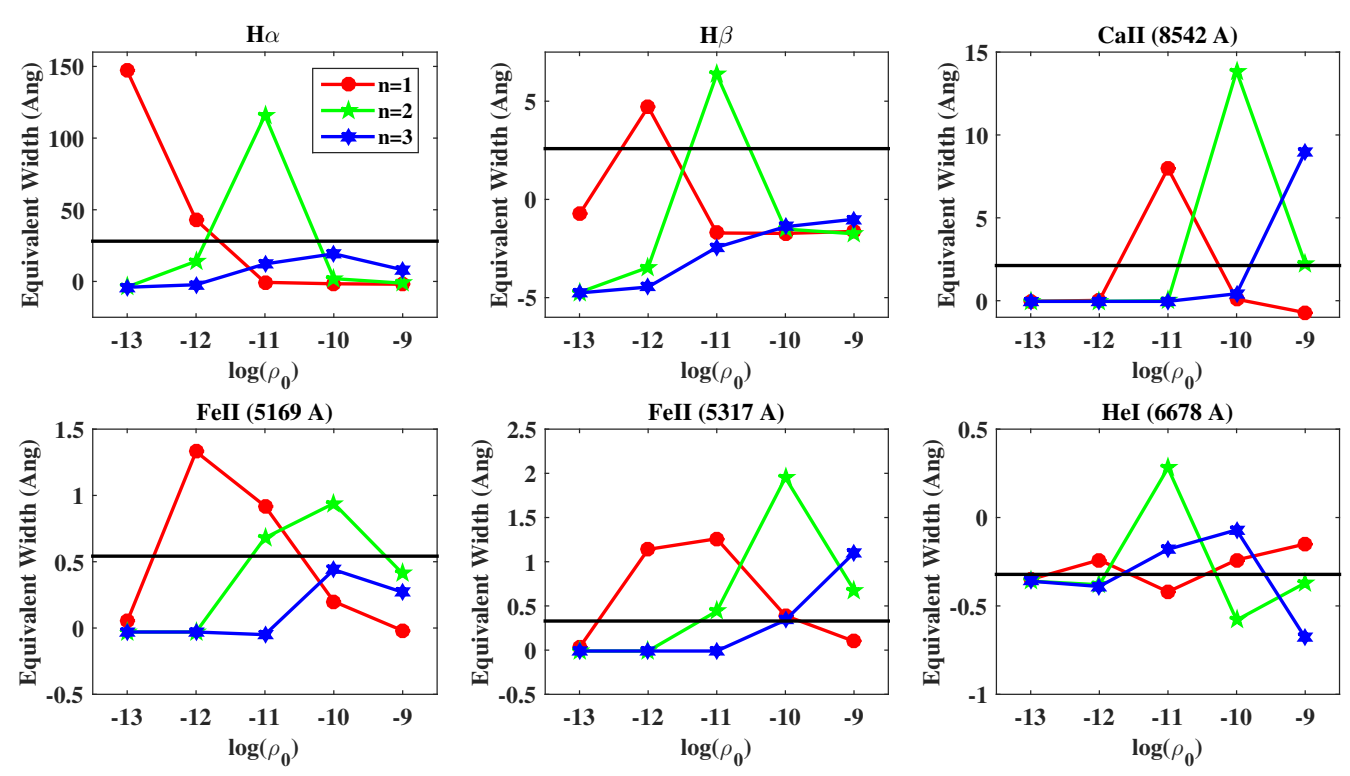

(b) Thick ES Turbulent disk model

Fig. 16.- Equivalent width $(\AA)$ of each individual line as a function of density $\left(\log \left(\rho_{0}\right)\right)$ for three different power law indices $n$ for models of radius $50 \mathrm{R}_{*}$ seen at $45^{\circ}$ inclination angle. The solid black line in each panel indicates the respective observed equivalent width. 